\title{
On Trajectory and Global Attractors for Semilinear Heat Equations with Fading Memory
}

\section{V.V. Chepyzhov \& A. Miranville}

\begin{abstract}
In this paper, we construct connected trajectory and global attractors for heat equations with linear fading memory and with nonlinear heat sources. No restriction on the polynomial growth of the nonlinear term is assumed. We also prove the existence of a global Lyapunov function for these equations under proper assumptions on the rate of exponential decay of the memory kernel. The existence of such a Lyapunov function implies that the trajectory and global attractors of the equation under consideration have a regular structure, i.e., they coincide with unstable trajectory sets issuing from the set of stationary points of the equation.
\end{abstract}

\section{INTRODUCTION}

In this paper, we study the following evolution integro-partial differential equation:

(0.1) $\partial_{t} u(x, t)=\Delta u(x, t)+\int_{0}^{\infty} k(s) \Delta u(x, t-s) d s-f(u(x, t))+g(x)$,

with boundary conditions $\left.u\right|_{\partial \Omega}=0$. The scalar function $u(x, t)$ depends on the spatial variable $x \in \Omega \Subset \mathbb{R}^{n}$ and the time $t \in \mathbb{R}$. In equation (0.1), $\Delta$ denotes the Laplace operator in $\mathbb{R}^{n}$. We also assume that $g(\cdot) \in L_{2}(\Omega)$ and that the nonlinear 
function $f(\cdot)$ belongs to $C^{1}(\mathbb{R})$ and satisfies the inequalities

$$
\begin{aligned}
& \text { (0.2) } \quad|f(u)| \leq \gamma_{0}\left(|u|^{p-1}+1\right), f(u) u \geq \gamma|u|^{p}-C, \quad p \geq 2 ; \gamma_{0}, \quad \gamma>0 ; \\
& \text { (0.3) } \quad f^{\prime}(u) \geq-D, \quad \forall u \in \mathbb{R} .
\end{aligned}
$$

The effects of memory in the equation are expressed through the linear time convolution of the function $\Delta u(\cdot)$ and the memory kernel $k(\cdot)$. We assume that $k(\cdot) \in C^{2}\left(\mathbb{R}_{+}\right), k(s) \geq 0, k^{\prime}(s) \leq 0$ for all $s \geq 0$, and $k(+\infty)=0$. Besides, we assume that the function $\mu(s) \stackrel{\text { def }}{\equiv}-k^{\prime}(s)$ satisfies the inequality

$$
\mu^{\prime}(s)+\delta \mu(s) \leq 0, \quad \forall s \geq 0,
$$

where $\delta$ is some positive number. It follows from these assumptions that the kernels $k(s)$ and $\mu(s)$ decay to zero with an exponential rate. This behavior reflects the fading of the far history in the model under consideration.

This model was proposed in [11]; similar equations were also considered in [16] and [17]. Equation (0.1) describes the heat flow in a rigid, isotropic, homogeneous heat conductor with memory in the presence of a nonlinear heat source. It is derived in the framework of the well-established theory of heat flows with memory due to Coleman and Gurtin (see [5]).

In $[7-10,18]$, the asymptotic behavior of the solutions of equation $(0.1)$ and of other related equations with memory was investigated by using the theory of dynamical systems and global attractors (see $[1,2,12,19])$. The authors of these papers used the so-called history space setting which suggests to consider some past history variables as additional components of the phase space corresponding to the equation under study. This fruitful idea was proposed by Dafermos (see [6]).

Let us shortly describe the history space setting for equation $(0.1)$ (see [7-10, $18]$ for more details). We introduce a new variable which reflects the past history of the equation, namely,

$$
\eta^{t}(x, s)=\int_{0}^{s} u(x, t-\tau) d \tau, \quad s \geq 0 .
$$

This function satisfies the following equation:

$$
\partial_{t} \eta^{t}(x, s)=u(x, t)-\partial_{s} \eta^{t}(x, s)
$$

(see Section 2). Integrating by parts in $s$ and using the assumption $k(+\infty)=0$, we rewrite the integral term in equation $(0.1)$ in the form

$$
\int_{0}^{\infty} k(s) \Delta u(x, t-s) d s=\int_{0}^{\infty} \mu(s) \Delta \eta^{t}(x, s) d s,
$$


where $\mu(s)=-k^{\prime}(s)$ (see Section 2). We write $u(t)=u(\cdot, t)$ and $\eta^{t}(\cdot, s)=$ $\eta^{t}(s)$. This choice of variables leads to the following system:

$$
\begin{aligned}
& \partial_{t} u(t)=\Delta u(t)+\int_{0}^{\infty} \mu(s) \Delta \eta^{t}(s) d s-f(u(t))+g, \\
& \partial_{t} \eta^{t}(s)=u(t)-\partial_{s} \eta^{t}(s),
\end{aligned}
$$

together with the boundary conditions $\left.u(t)\right|_{\partial \Omega}=0$ and $\left.\eta^{t}(s)\right|_{\partial \Omega}=0$ for all $t$, $s \geq 0$. We supplement system (0.7)-(0.8) with the initial data

$$
\begin{aligned}
\left.u\right|_{t=0} & =u_{0}, \\
\left.\eta^{t}(s)\right|_{t=0} & =\eta_{0}(s), \quad s \geq 0 .
\end{aligned}
$$

We assume that the functions $u_{0}$ and $\eta_{0}$ are quite arbitrary and belong to some function spaces, e.g., $u_{0} \in L_{2}(\Omega)$ and $\eta_{0} \in L_{2, \mu}\left(\mathbb{R}_{+} ; H_{0}^{1}(\Omega)\right)$, where $L_{2, \mu}:=$ $L_{2, \mu}\left(\mathbb{R}_{+} ; H_{0}^{1}(\Omega)\right)$ is the corresponding weighted (for the variable $s$ ) Hilbert space with weight $\mu(s), s \geq 0$ (see, e.g., [9]).

Now, setting

$$
z(t)=\left(u(t), \eta^{t}\right), \quad z_{0}=\left(u_{0}, \eta_{0}\right)
$$

and

$$
\begin{aligned}
& \mathcal{L}(z)=\left(\Delta u+\int_{0}^{\infty} \mu(s) \Delta \eta(s) d s, u-\partial_{s} \eta\right), \\
& \mathcal{G}(z)=(-f(u)+g, 0),
\end{aligned}
$$

problem (0.7)-(0.10) takes the compact form

$$
\begin{aligned}
& \partial_{t} z=\mathcal{L} z+\mathcal{G}(z), \\
& \left.z\right|_{t=0}=z_{0} .
\end{aligned}
$$

If one can prove that the Cauchy problem $(0.11)$ and (0.12) has a unique solution $z(t), t \geq 0$, with values in a suitable phase space $\mathcal{H}$ (e.g., in $\mathcal{H}=$ $\left.L_{2}(\Omega) \times L_{2, \mu}\right)$, then one can construct a semigroup of operators $S(t): \mathcal{H} \rightarrow \mathcal{H}$, $S(t) z_{0}=z(t), t \geq 0$, and study the global attractor of this autonomous dynamical system. This scheme is called the history space setting and it was successfully applied to various equations with memory in $[7-10,18]$ and in many other papers. Unfortunately, this approach leads to a rather complicated phase space $\mathcal{H}$ for the corresponding dynamical system. It is also difficult to study properties of the semigroup $\{S(t)\}$ such as continuity, compactness, or asymptotic compactness. These obstacles often lead to various additional restrictions on the terms of 
the equation with memory under study (e.g., restrictions on the growth $p$ (see $(0.2)$ ) of the nonlinear function $f(u)$ in $(0.1))$ which seem unnecessary.

In the present paper, we study the behavior of the solutions of equation (0.1) as $t \rightarrow+\infty$ by constructing the so-called trajectory attractor of this equation. This method was proposed in [2,3] and it was successfully applied by the authors in [4] to the study of dissipative semilinear hyperbolic equations with memory.

We assume that the function $u(x, t)$ in equation (0.1) is known for all $t \leq 0$ and $u(x, t)$ does not necessarily satisfy the equation for negative $t$. This assumption can be reflected in the "initial condition" which we write in the form

$$
\left.u\right|_{t \leq 0}=v(x, t), \quad t \leq 0,
$$

where the function $v(t)=v(\cdot, t), t \leq 0$, can be chosen quite arbitrarily. We only assume that $v(\cdot) \in \mathcal{E}^{-}=C_{b}\left(\mathbb{R}_{-} ; L_{2}(\Omega)\right) \cap L_{2}^{b}\left(\mathbb{R}_{-} ; H_{0}^{1}(\Omega)\right)$, where $\mathbb{R}_{-}=$ $(-\infty, 0]$.

We are looking for a function $u(t)=u(\cdot, t), t \in \mathbb{R}$, belonging to the space $C\left(\mathbb{R} ; L_{2}(\Omega)\right) \cap L_{2}^{b}\left(\mathbb{R} ; H_{0}^{1}(\Omega)\right)$ such that $u(t)$ coincides with $v(t)$ for all $t \leq 0$ and, for $t \geq 0, u(t)$ satisfies equation $(0.1)$.

In Section 2, we prove that problem (0.1) and (0.13) has a unique solution for every $v \in \mathcal{E}^{-}$(a similar theorem is proved in [9]).

We denote by $v(s), s \leq 0$, the elements of the space $\mathcal{E}^{-}$, where $s$ stands for the time variable in place of $t$.

For every fixed $t \geq 0$, we consider the mapping $S(t): \mathcal{E}^{-} \rightarrow \mathcal{E}^{-}$acting by the formula

$$
(S(t) v)(s)=u(t+s), \quad \forall s \leq 0,
$$

where $u(t), t \in \mathbb{R}$, is the solution of problem $(0.1)$ and (0.13) with initial datum $v \in \mathcal{E}^{-}$. It is easy to see that the family of operators $\{S(t) \mid t \geq 0\}$ forms a semigroup in $\mathcal{E}^{-}$.

Our aim is then to construct and to study the trajectory and global attractors of the semigroup $\{S(t)\}$ in $\mathcal{E}^{-}$. In Section 1, we present a general scheme for the construction of the trajectory and global attractors for equations with memory.

Recall that, in order to define the global attractor, we have to specify a topology in the space $\mathcal{E}^{-}$, which will be used to measure the attraction of the bounded sets of initial data from $\mathcal{E}^{-}$under the action of $S(t)$ as $t$ tends to infinity. For this purpose, we take the local uniform convergence topology of the space $C^{\text {loc }}\left(\mathbb{R}_{-} ; E\right)$. Here, we take $E=L_{2}(\Omega)$. To define the bounded sets in $\mathcal{E}^{-}$, we use the norm of the space $\mathcal{E}^{-}$itself (see Sections 1 and 4). We shall call the corresponding global attractor of the semigroup $\{S(t)\}$ the trajectory attractor since the semigroup $\{S(t)\}$ acts in the trajectory space $\mathcal{E}^{-}$.

Definition 0.1. A set $\mathfrak{A} \subset \mathcal{E}^{-}$is called the trajectory $\left(\mathcal{E}^{-}, C^{\text {loc }}\left(\mathbb{R}_{-} ; E\right)\right)$ attractor of the semigroup $\{S(t)\}$ generated by problem $(0.1)$ and $(0.13)$ if 
(1) the set $\mathfrak{A}$ is bounded in $\mathcal{E}^{-}$and compact in $C^{\text {loc }}\left(\mathbb{R}_{-} ; E\right)$;

(2) the set $\mathfrak{A}$ is strictly invariant with respect to $\{S(t)\}$, that is,

$$
S(t) \mathfrak{A}=\mathfrak{A}, \quad \forall t \geq 0 ;
$$

(3) the set $\mathfrak{A}$ is an attracting (in $C^{\text {loc }}\left(\mathbb{R}_{-} ; E\right)$ ) set of the semigroup $\{S(t)\}$ : for any bounded set $B \subset \mathcal{E}^{-}$, the set $S(t) B$ converges to $\mathfrak{A}$ as $t \rightarrow+\infty$ in the topology of $C^{\text {loc }}\left(\mathbb{R}_{-} ; E\right)$, i.e., for every $M>0$,

$$
\sup _{v \in B} \operatorname{dist}_{C([-M, 0] ; E)}(S(t) v, \mathfrak{A}) \rightarrow 0 \quad(t \rightarrow+\infty) .
$$

In Sections 3 and 4, we prove our main result.

Theorem 0.2. Under assumptions (0.2), (0.3), and (0.4), the semigroup $\{S(t)\}$ of problem $(0.1)$ and $(0.13)$ possesses the trajectory $\left(\mathcal{E}^{-}, C^{\text {loc }}\left(\mathbb{R}_{-} ; E\right)\right)$-attractor $\mathfrak{A}$. Furthermore, the set $\mathfrak{A}$ is connected in the space $C^{\text {loc }}\left(\mathbb{R}_{-} ; E\right)$ (see also Section 5).

We note that, due to the strict invariance property, the trajectory attractor $\mathfrak{A}$ consists of functions $v(t), t \leq 0$, which satisfy equation (0.1) for all $t \leq 0$.

We then use the trajectory attractor $\mathfrak{A}$ of the equation with memory $(0.1)$ to construct the global attractor (in $E=L_{2}(\Omega)$ ) of this equation. For any set $B \subset \mathcal{E}^{-}$, we set

$$
B(h)=\{v(h) \mid v \in B\} \subset E,
$$

where $h \leq 0$ is an arbitrary fixed number. In particular, we set

$$
\mathfrak{A}(h)=\{v(h) \mid v \in \mathfrak{A}\} \subset E .
$$

The set $\mathfrak{A}(h)$ is independent of $h$, i.e., $\mathfrak{A}(h)=\mathfrak{A}(0)$ for all $h \leq 0$, since $\mathfrak{A}$ is strictly invariant w.r.t. $\{S(t)\}$.

Definition 0.3. A set $\mathcal{A} \subset E$ is called the global $\left(\mathcal{E}^{-}, E\right)$-attractor of equation $(0.1)$ if

(1) the set $\mathcal{A}$ is compact in $E=L_{2}(\Omega)$;

(2) the set $\mathcal{A}$ attracts the bounded solutions of (0.1), that is, for any bounded set $B$ from $\mathcal{E}^{-}$

$$
\sup _{v \in B} \operatorname{dist}_{E}(u(t, v), \mathcal{A}) \rightarrow 0 \quad(t \rightarrow+\infty),
$$

where $u(t, v)$ is the solution of $(0.1)$ with initial datum $v$;

(3) the set $\mathcal{A}$ is the minimal compact attracting set which satisfies (0.16).

We prove in Section 4 that the set $\mathcal{A}=\mathfrak{A}(0)$ is the global $\left(\mathcal{E}^{-}, E\right)$-attractor of equation (0.1) and the set $\mathcal{A}$ is connected (see Section 5). In Section 5, we also establish some additional smoothness properties of the trajectory and global attractors of equation (0.1). 
In Section 6, we consider the problem of the regularity of the trajectory and global attractors of the heat equation with memory (0.1). It is well-known that the semilinear heat equation without memory (corresponding to $k \equiv 0$ in $(0.1)$ ) possesses the regular global attractor (see $[1,12])$. This assertion follows from the existence of a global Lyapunov function for this equation. However, we show in Section 6 that it is impossible to construct a Lyapunov function for the equation with memory ( 0.1$)$ by using the method known for the equation without memory. Nevertheless, we construct in Section 6 another Lyapunov function under some additional restrictions on the nonlinear function $f(u)$ and the memory kernel $k$. We prove the following result.

Theorem 0.4. If the number $D$ from inequality (0.3) satisfies

$$
D<\lambda_{1}+\delta,
$$

where $\lambda_{1}$ is the first eigenvalue of the operator $-\Delta$ with zero boundary conditions and $\delta$ is taken from (0.4), then the semigroup $\{S(t)\}$ of problem (0.1) and (0.13) has a global Lyapunov function in the space $\mathcal{E}^{-}$.

Having a global Lyapunov function, we prove that the trajectory and global attractors of $(0.1)$ have a regular structure. We denote by $\mathcal{N}$ the set of all stationary solutions of $(0.1)$, i.e., the functions $z(\cdot) \in H_{0}^{1}(\Omega)$ such that

$$
(1+\alpha) \Delta z(x)-f(z(x))+g(x)=0,\left.\quad z\right|_{\partial \Omega}=0,
$$

where $\alpha=\int_{0}^{\infty} k(s) d s$.

Definition 0.5. The following set is called the unstable trajectory set issuing from $\mathcal{N}$ :

$\mathcal{M}^{+}(\mathcal{N})=\left\{v(\cdot) \in \mathcal{E}^{-} \mid v(t)\right.$ satisfies $(0.1)$ for $t \leq 0$,

$$
\text { and } \left.\operatorname{dist}_{E}(v(t), \mathcal{N}) \rightarrow 0(t \rightarrow-\infty)\right\} .
$$

The set $\mathcal{M}^{+}(\mathcal{N})$ is strictly invariant w.r.t. $\{S(t)\}$ and, besides, $\mathcal{M}^{+}(\mathcal{N}) \subseteq \mathfrak{A}$. In Section 6, we prove the following result.

Theorem 0.6. Under condition (0.17), we have

$$
\mathfrak{A}=\mathcal{M}^{+}(\mathcal{N}) \quad \text { and } \quad \mathcal{A}=\mathcal{M}^{+}(\mathcal{N})(0) .
$$

If the set $\mathcal{N}$ is finite, i.e., $\mathcal{N}=\left\{z_{1}, z_{2}, \ldots, z_{m}\right\}$, then

$$
\mathfrak{A}=\bigcup_{j=1}^{m} \mathcal{M}^{+}\left(z_{j}\right) \text { and } \mathcal{A}=\bigcup_{j=1}^{m} \mathcal{M}^{+}\left(z_{j}\right)(0) .
$$

Furthermore, in that case, for any solution $u(t)$ of equation (0.1), there exists a stationary solution $z_{j}$ such that $\left\|u(t)-z_{j}\right\|_{E} \rightarrow 0(t \rightarrow+\infty)$. 
Finally, we emphasize that it is quite reasonable to expect that the past history setting from $[7-10,18]$ and the trajectory attractor approach of the present paper (see also [4]) should be, in some proper sense, related. In particular, the corresponding global attractor and trajectory attractor should be homeomorphic in the corresponding spaces. In a forthcoming paper, we intend to compare these two methods of study of evolution equations with memory in detail and to establish the precise relation between their global and trajectory attractors. It would also be important to study the existence of a Lyapunov function in the past history setting in order to study the structure of the corresponding global attractors.

\section{Equations With MEMORY and Their Trajectory and GLOBAL ATTRACTORS}

We consider the following abstract evolution equation:

$$
\partial_{t} u(t)=A\left(u(t), u^{t}(\cdot)\right), \quad t \geq 0 .
$$

We assume that the function $u(t)$ is known for all $t \leq 0$ and our problem is to continue $u(t)$ for $t \geq 0$ in such a way that $u(t)$ satisfies equation (1.1). The operator $A(\cdot, \cdot)$ in the right-hand side of $(1.1)$ depends on two parameters. The first parameter is the value of the function $u$ at time $t$, while the second one is the entire function $u\left(t^{\prime}\right)$ for all $t^{\prime} \leq t$. In (1.1), we denote by $u^{t}(\cdot)$ the function $u\left(t^{\prime}\right), t^{\prime} \leq t$, with range $(-\infty, t]$.

Thus, the derivative $\partial_{t} u(\cdot)$ at time $t$ depends not only on the value $u(t)$ of the function $u$ at this time $t$, but also on all the values $u\left(t^{\prime}\right)$ for $t^{\prime} \leq t$. This reflects the appearance of memory effects in the evolution equation. Recall that, in the usual partial differential equations, the time derivative $\partial_{t} u(t)$ depends only on $u(t)$, that is, the right-hand side $A$ in (1.1) has the form $A(u(t))$. In the next section, we shall consider an example of evolution equation of the form (1.1).

Here, we discuss a general approach for the study of the equation with memory (1.1). We assume that the function $u$ takes values in a Banach space $E$, i.e., $u(t) \in E$ for all $t \in \mathbb{R}$. We note that the function $u(t)$ does not necessarily satisfy equation (1.1) for negative $t$. Therefore, we may choose $u(t)$ for $t \leq 0$ quite arbitrarily. We can write this circumstance as an "initial datum" for equation (1.1) of the form

$$
\left.u\right|_{t \leq 0}=v(t), \quad t \leq 0,
$$

where the function $v(\cdot)$ belongs to some class of functions $\mathcal{E}^{-}$, with range $(-\infty, 0]$ and with values in the space $E$. We shall specify the space $\mathcal{E}^{-}$below.

Our aim is to study the asymptotic behavior of the solutions of problem (1.1) and (1.2) as $t \rightarrow+\infty$. To construct the dynamical system or the semigroup corresponding to this problem, we make the following basic assumption. We assume that problem (1.1) and (1.2) has a unique solution for any initial datum $v(\cdot)$. We note that this question can be the subject of a separate deep investigation for a 
given nonlinear equation of the form (1.1), where one has to define precisely the notion of a solution of (1.1) and (1.2) and to specify a class of functions in which the solutions live.

We denote by $C_{b}\left(\mathbb{R}_{-} ; E\right)$ the space of bounded continuous functions with range $\mathbb{R}_{-}=(-\infty, 0]$ and with values in $E$. We consider a vector subspace $\mathcal{E}^{-} \subseteq$ $C_{b}\left(\mathbb{R}_{-} ; E\right)$. The subspace $\mathcal{E}^{-}$serves as the phase space of the dynamical system that we are constructing. We denote by $v(s), s \leq 0$, the elements of $\mathcal{E}^{-}$, replacing the time variable $t$ by $s$. We clarify our main assumption: for every $v \in \mathcal{E}^{-}$, problem (1.1) and (1.2) has a unique solution $u(\cdot)$ belonging to the space $C_{b}(\mathbb{R} ; E)$, that is, $u(t)=v(t)$ for all $t \leq 0$ and $u(t)$ satisfies equation (1.1) for all $t \geq 0$. Moreover, we assume that the function $u_{h}(s)=u(h+s), s \leq 0$, also belongs to $\mathcal{E}^{-}$for all $h \geq 0$.

For every fixed $t \geq 0$, we consider the following mapping acting in $\mathcal{E}^{-}$:

$$
(S(t) v)(s)=u(t+s), \quad s \leq 0,
$$

where $u(t)$ is the solution of (1.1) and (1.2) with initial datum $v(\cdot)$. It follows from our assumptions that $S(t) v \in \mathcal{E}^{-}$for every $t \geq 0$. Then, clearly, the family of mappings $\{S(t) \mid t \geq 0\}=\{S(t)\}$ forms a semigroup acting in $\mathcal{E}^{-}$, i.e., $S(0)$ is the identity operator and $S\left(t_{1}+t_{2}\right)=S\left(t_{1}\right) \circ S\left(t_{2}\right)$ for all $t_{1}, t_{2} \geq 0$.

Remark 1.1. In fact, the mappings $S(t)$ are also well defined for $t \leq 0$ and we obtain the group $\{S(t) \mid t \in \mathbb{R}\}$. We only have to note that, for $t \leq 0$, the mappings $S(t)$ are independent of the solutions of equation (1.1).

We study the global attractor of the semigroup $\{S(t)\}$ acting in the phase space $\mathcal{E}^{-}$. It is known that, in order to define a global attractor, we have to determine a topology on $\mathcal{E}^{-}$and a collection of bounded sets in $\mathcal{E}^{-}$. We first consider the local uniform convergence topology $C^{\text {loc }}\left(\mathbb{R}_{-} ; E\right)$. Recall that a sequence of functions $\left\{f_{n}\right\} \subset \mathcal{E}^{-}$converges to a function $f \in \mathcal{E}^{-}$if, for every $M \geq 0$,

$$
\max _{s \in[-M, 0]}\left\|f_{n}(s)-f(s)\right\|_{E} \rightarrow 0 \quad(s \rightarrow+\infty) .
$$

It is well-known that the space $C^{\text {loc }}\left(\mathbb{R}_{-} ; E\right)$ is metrizable and the corresponding metric space is complete. Nevertheless, in order to define the bounded sets in $\mathcal{E}^{-}$, we shall use another metric. For example, this metric can be generated by the norm of the space $C_{b}\left(\mathbb{R}_{-} ; E\right)$, i.e.,

$$
\|f\|_{C_{b}\left(\mathbb{R}_{-} ; E\right)}=\sup _{s \leq 0}\|f(s)\|_{E} .
$$

Another possibility is the following metric. Let $E_{1}$ be a Banach space such that $E_{1} \subseteq E$. Let $\mathcal{E}^{-} \subset L_{p}^{b}\left(\mathbb{R}_{-} ; E_{1}\right)(p \geq 1)$. The norm of the space $L_{p}^{b}\left(\mathbb{R}_{-} ; E_{1}\right)$ reads

$$
\|f\|_{L_{p}^{b}\left(\mathbb{R}_{-} ; E_{1}\right)}=\left(\sup _{t \leq 0} \int_{t-1}^{t}\|f(s)\|_{E_{1}}^{p} d s\right)^{1 / p} .
$$


Then, the desired metric is generated by the norm of the space $C_{b}\left(\mathbb{R}_{-} ; E\right) \cap$ $L_{p}^{b}\left(\mathbb{R}_{-} ; E_{1}\right)$, i.e., by the sum

$$
\|f\|_{C_{b}\left(\mathbb{R}_{-} ; E\right)}+\|f\|_{L_{p}^{b}\left(\mathbb{R}_{-} ; E_{1}\right)} .
$$

We actually assume that $\mathcal{E}^{-} \subseteq \mathcal{F}$, where $\mathcal{F}$ is a Banach space whose norm $\|\cdot\|_{\mathcal{F}}$ will be used to define the bounded sets in $\mathcal{E}^{-}$. We assume that the norm of $\mathcal{F}$ satisfies the following inequality on $\mathcal{E}^{-}$:

$$
\|S(h) v\|_{\mathcal{F}}=\|v(h+\cdot)\|_{\mathcal{F}} \leq\|v(\cdot)\|_{\mathcal{F}}, \quad \forall h \leq 0, \forall v \in \mathcal{E}^{-} .
$$

This property looks very natural and is obviously valid for the above examples (i.e., when $\mathcal{F}=C_{b}\left(\mathbb{R}_{-} ; E\right)$ and $\mathcal{F}=C_{b}\left(\mathbb{R}_{-} ; E\right) \cap L_{p}^{b}\left(\mathbb{R}_{-} ; E_{1}\right)$ ).

We first define the notion of an attracting set for the semigroup $\{S(t)\}$ in $\mathcal{E}^{-}$.

Definition 1.2. A set $P \subset \mathcal{E}^{-}$is called attracting (in $C^{\text {loc }}\left(\mathbb{R}_{-} ; E\right)$ ) for the semigroup $\{S(t)\}$ if, for any set $B \subset \mathcal{E}^{-}$, bounded in $\mathcal{F}$, and for any neighborhood $\mathcal{O}(P)$ in $C^{\text {loc }}\left(\mathbb{R}_{-} ; E\right)$ of the set $P$, there exists a number $t_{1}=t_{1}(B, \mathcal{O})$ such that $S(t) B \subseteq \mathcal{O}(P)$ for all $t \geq t_{1}$. This is equivalent to the following: for any $M>0$,

$$
\operatorname{dist}_{C([-M, 0] ; E)}(S(t) B, P) \rightarrow 0 \quad(t \rightarrow+\infty) .
$$

Here, $\operatorname{dist}_{\mathcal{M}}(A, B)$ denotes the Hausdorff semi-distance from a set $A$ to a set $B$ in a metric space $\mathcal{M}$ with metric $\rho_{\mathcal{M}}(\cdot, \cdot)$, that is,

$$
\operatorname{dist}_{\mathcal{M}}(A, B)=\sup _{a \in A} \inf _{b \in B} \rho_{\mathcal{M}}(a, b) .
$$

We now define the global attractor of the semigroup $\{S(t)\}$, which we call the trajectory attractor since this semigroup acts in the space of (semi-)trajectories $\mathcal{E}^{-}$.

Definition 1.3. A set $\mathfrak{A} \subset \mathcal{E}^{-}$is called the trajectory $\left(\mathcal{F}, C^{\text {loc }}\left(\mathbb{R}_{-} ; E\right)\right)$-attractor of problem (1.1) and (1.2) if

(1) the set $\mathfrak{A}$ is bounded in $\mathcal{F}$ and compact in $C^{\text {loc }}\left(\mathbb{R}_{-} ; E\right)$;

(2) the set $\mathfrak{A}$ is strictly invariant with respect to $\{S(t)\}$, that is, $S(t) \mathfrak{A}=\mathfrak{A}$ for all $t \geq 0$

(3) the set $\mathfrak{A}$ is an attracting (in $C^{\text {loc }}\left(\mathbb{R}_{-} ; E\right)$ ) set of the semigroup $\{S(t)\}$.

Remark 1.4. Notice that the notion of a global $(F, D)$-attractor of a semigroup was introduced in [1]. Here, $F$ denotes a metric space taken to define the bounded sets and $D$ is a topological space in which one measures the attraction of bounded sets to the attractor.

Following [1] and [2], we formulate the main theorem on the existence of the trajectory attractor of problem (1.1) and (1.2).

Recall that the semigroup $\{S(t)\}$ is called uniformly bounded in $\mathcal{F}$ if, for any set $B \subset \mathcal{E}^{-}$, bounded in $\mathcal{F}$, there exists a bounded set $B_{1}$ such that $S(t) B \subseteq B_{1}$ for all $t \geq 0$. 
Theorem 1.5. Let the semigroup $\{S(t)\}$ acting in $\mathcal{E}^{-} \subseteq C_{b}\left(\mathbb{R}_{-} ; E\right)$ and corresponding to problem (1.1) and (1.2) be uniformly bounded in $\mathcal{F}$ and have an attracting set $P \subset \mathcal{E}^{-}$. We further assume that the set $P$ is bounded in $\mathcal{F}$ and compact in $C^{\text {loc }}\left(\mathbb{R}_{-} ; E\right)$. Then, there exists the trajectory $\left(\mathcal{F}, C^{\text {loc }}\left(\mathbb{R}_{-} ; E\right)\right)$-attractor $\mathfrak{A}$ of the semigroup $\{S(t)\}$ and $\mathfrak{A}=\omega(P)$, where

$$
\omega(P)=\bigcap_{h \geq 0}\left[\bigcup_{t \geq h} S(t) P\right]_{C^{\operatorname{loc}}\left(\mathbb{R}_{-} ; E\right)}
$$

is the $\omega$-limit set of $P$ w.r.t. $\{S(t)\}$ in $C^{\text {loc }}\left(\mathbb{R}_{-} ; E\right)$.

Proof. We consider the spaces $C_{b}(\mathbb{R} ; E)$ and $C^{\text {loc }}(\mathbb{R} ; E)$. We denote by $\mathcal{K}^{+}$ the space of all solutions of equation (1.1) with all possible initial data $v \in \mathcal{E}^{-}$, that is,

$$
\mathcal{K}^{+}=\left\{u(t), t \in \mathbb{R} \mid\left\{\begin{array}{ll}
u(t)=v(t), & t \leq 0, v \in \mathcal{E}^{-} \\
u(t) \text { satisfies (1.1), } & t \geq 0, v \in \mathcal{E}^{-}
\end{array}\right\} .\right.
$$

It is clear that $\mathcal{K}^{+} \subset C_{b}(\mathbb{R} ; E)$. The translation semigroup $\{T(h)\}=\{T(h) \mid$ $h \geq 0$ \} acts on $\mathcal{K}^{+}$by the formula

$$
T(h) u(t)=u(t+h), \quad h \geq 0 .
$$

We now construct the global $\left(\mathcal{F}, C^{\text {loc }}(\mathbb{R} ; E)\right)$-attractor of the semigroup $\{T(h)\}$. We extend the metric of $\mathcal{F}$ to the space $\mathcal{K}^{+}$. The extended metric measures the distance between the "tails" $u(t), t \leq 0$, in $\mathcal{F}$. This new metric is well defined on $\mathcal{K}^{+}$since $u(t)$, for $t \geq 0$, is uniquely determined by the values $u(t)=v(t)$ for $t \leq 0$. It follows easily that the semigroup $\{T(h)\}$ is continuous in the topology $C^{\text {loc }}(\mathbb{R} ; E)$. Consider the set $P^{+} \subset \mathcal{K}^{+}$defined by

$$
P^{+}=\left\{u(t), t \in \mathbb{R} \mid\left\{\begin{array}{ll}
u(t)=v(t), & t \leq 0, v \in P \\
u(t) \text { satisfies (1.1), } & t \geq 0, v \in P
\end{array}\right\} .\right.
$$

The set $P$ is bounded in $\mathcal{F}$. It is clear that the set $P^{+}$is also bounded in $\mathcal{F}$, since the semigroup $\{S(t)\}$ is uniformly bounded in $\mathcal{F}$. Furthermore, the set $P^{+}$is precompact in $C^{\text {loc }}(\mathbb{R} ; E)$, since $P$ is compact in $C^{\text {loc }}\left(\mathbb{R}_{-} ; E\right)$. Finally, the set $P^{+}$ is attracting for the semigroup $\{T(h)\}$. Therefore, the theorem from [1] on the existence of the global $(F, D)$-attractor of a continuous semigroup is applicable to the semigroup $\{T(h)\}$ acting in $\mathcal{K}^{+}$(see also [2] and [4]). We set $F=\mathcal{F}$ and $D=C^{\text {loc }}(\mathbb{R} ; E)$. We denote by $\mathfrak{A}^{+}=\omega\left(P^{+}\right)$the corresponding global $(F, D)$ attractor of the semigroup $\{T(h)\}$. We now restrict the set $\mathfrak{A}^{+}$to the negative semiaxis $\mathbb{R}_{-}=(-\infty, 0]$. We denote by $\mathfrak{A}$ the set so constructed. This set satisfies all the properties of the definition of the trajectory attractor. Moreover, we have $\mathfrak{A}=\omega(P)$, where the $\omega$-limit set of $P$ is taken in the topology of $C^{\text {loc }}\left(\mathbb{R}_{-} ; E\right)$. We leave the details to the reader. 
Notice that the continuity in $C^{\text {loc }}\left(\mathbb{R}_{-} ; E\right)$ of the semigroup $\{S(t)\}$ is not assumed in Theorem 1.5. In the proof, we have only used the natural continuity of the extended translation semigroup $\{T(h)\}$. This approach was also used in $[2,3]$ in order to construct trajectory attractors for evolution equations without uniqueness such as the 3D Navier-Stokes system.

Corollary 1.6. If $v(\cdot) \in \mathfrak{A}$, then the corresponding solution $u(t), t \in \mathbb{R}$, of equation (1.1) satisfies this equation for all $t \in \mathbb{R}$.

Proof. We apply the strict invariance property of the trajectory attractor (see point (2) in Definition 1.3). For a given $v(\cdot) \in \mathfrak{A}$, we consider the corresponding solution $u(t)$. Since $\mathfrak{A}$ is strictly invariant with respect to $\{S(t)\}$, then, for every $h>0$, we can find a function $v_{h}(\cdot) \in \mathfrak{A}$ (and the corresponding solution $u_{h}(\cdot)$ ) such that $S(h) v_{h}=v$, that is, $u_{h}(h+s)=u(s)$ for all $s \leq 0$, but also, by uniqueness, for all $s \in \mathbb{R}$. The function $u_{h}(t)$ satisfies (1.1) for $t \geq 0$. Therefore, $u(t)$ satisfies (1.1) for $t \geq-h$. Letting $h \rightarrow+\infty$, we deduce that $u(t)$ satisfies (1.1) for all $t \in \mathbb{R}$.

We now formulate a useful compactness criterion in the space $C^{\text {loc }}\left(\mathbb{R}_{-} ; E\right)$.

Lemma 1.7. A set $B \subset C^{\text {loc }}\left(\mathbb{R}_{-} ; E\right)$ is compact in $C^{\text {loc }}\left(\mathbb{R}_{-} ; E\right)$ if and only if it is compact in $C([-M, 0] ; E)$ for every $M>0$.

We shall use this criterion together with the following sufficient condition for the compactness in $C([-M, 0] ; E)$.

Lemma 1.8. Let $E_{1} \Subset E \subset E^{\prime}$ be three Banach spaces and let the first embedding be compact. We fix numbers $p>1$ and $M>0$. Let a set $B$ be bounded in the space $L_{\infty}\left(-M, 0 ; E_{1}\right)$ and let the set $B^{\prime}=\left\{\partial_{t} u \mid u \in B\right\}$ be bounded in the space $L_{p}\left(-M, 0 ; E^{\prime}\right)$. Here, $\partial_{t} u=\partial_{t} u(t)$ denotes the derivative of the function $u=u(t)$ in the sense of distributions of the space $\mathcal{D}^{\prime}\left(-M, 0 ; E^{\prime}\right)($ see $[14])$. Then, the set $B$ is precompact in $C([-M, 0] ; E)$.

For the proof, see, for instance, [2].

We now show how to construct the global attractor in the space $E$ for the equation with memory $(1.1)$, having the trajectory attractor in the space $C^{\text {loc }}\left(\mathbb{R}_{-} ; E\right)$.

For any set $B \subset \mathcal{E}^{-} \subseteq C_{b}\left(\mathbb{R}_{-} ; E\right)$, we set

$$
B(h)=\{u(h) \mid u \in B\} \subset E,
$$

where $h \leq 0$ is fixed. In particular, we consider the following set belonging to $E$ :

$$
\mathfrak{A}(h)=\{u(h) \mid u \in \mathfrak{A}\} .
$$

Proposition 1.9. The set $\mathfrak{A}(h)$ is independent of $h$, i.e., $\mathfrak{A}(h)=\mathfrak{A}(0)$, for all $h \leq 0$.

This fact is a direct consequence of the invariance property of the trajectory attractor. We now define the global attractor (in $E$ ) for equation (1.1). 
Definition 1.10. A set $\mathcal{A} \subset E$ is called the global $(\mathcal{F}, E)$-attractor of equation (1.1) if

(1) the set $\mathcal{A}$ is compact in $E$;

(2) the set $\mathcal{A}$ attracts the bounded (in $\mathcal{F}$ ) solutions of (1.1), that is, for any bounded set $B$ from the space $\mathcal{E}^{-}$,

$$
\operatorname{dist}_{E}((S(t) B)(0), \mathcal{A}) \rightarrow 0 \quad(t \rightarrow+\infty) ;
$$

(3) the set $\mathcal{A}$ is the minimal compact attracting set, that is, if a set $\mathcal{A}^{\prime}$ is compact in $E$ and satisfies (1.11) (with $\mathcal{A}^{\prime}$ in place of $\mathcal{A}$ ), then $\mathcal{A} \subseteq \mathcal{A}^{\prime}$.

We deduce from (1.11) that, for any bounded set of initial data $B=\{v \mid$ $\left.\|v\|_{\mathcal{F}} \leq R\right\}$, the corresponding solutions $u(t)=u(t, v)$ tend to $\mathcal{A}$ in the norm of $E$ as $t \rightarrow+\infty$ uniformly w.r.t. $v \in B$, that is,

$$
\sup _{v \in B} \operatorname{dist}_{E}(u(t, v), \mathcal{A}) \rightarrow 0 \quad(t \rightarrow+\infty) .
$$

Theorem 1.11. Under the assumptions of Theorem 1.5, the set

$$
\mathcal{A}=\mathfrak{A}(0)
$$

is the global $(\mathcal{F}, E)$-attractor of problem (1.1) and (1.2).

The proof is given in [4] and follows from the properties of the trajectory attractor $\mathfrak{A}$.

In the next sections, we shall consider semilinear heat equations with memory and we shall construct the trajectory and global attractors for these equations.

\section{Semilinear Heat Equations With Memory}

We consider the following parabolic equation with memory in a bounded domain $\Omega \Subset \mathbb{R}^{n}:$

$$
\partial_{t} u(x, t)=\Delta u(x, t)+\int_{0}^{\infty} k(s) \Delta u(x, t-s) d s-f(u(x, t))+g(x),
$$

Here, $u(x, t)$ is a scalar unknown function of the variables $x \in \Omega$ and $t \geq 0$, and $\Delta=\Delta_{x}$ is the Laplace operator in $\mathbb{R}^{n}$. The nonlinear scalar function $f(u)$, $u \in \mathbb{R}$, belongs to $C^{1}(\mathbb{R})$ and satisfies the following inequalities:

$$
\begin{array}{ll}
f^{\prime}(u) \geq-D ; & \\
f(u) u \geq \gamma|u|^{p}-C, & p \geq 2, \gamma>0 ; \\
|f(u)| \leq \gamma_{0}\left(|u|^{p-1}+1\right), & \gamma_{0}>0, \forall u \in \mathbb{R} .
\end{array}
$$


The function $g(x)$ belongs to $L_{2}(\Omega)$.

Concerning the memory kernel $k(\cdot)$, we assume that $k(\cdot) \in C^{2}\left(\mathbb{R}_{+}\right), k(s) \geq$ 0 for all $s \geq 0$, and

$$
\begin{aligned}
\mu^{\prime}(s) & \stackrel{\text { def }}{\equiv}-k^{\prime}(s) \\
\mu^{\prime}(s)+\delta \mu(s) & \leq 0, \quad \forall s \geq 0(\delta>0) .
\end{aligned}
$$

It follows from (2.6) that the positive function $k(s)$ is decreasing. We also assume that

$$
k(+\infty)=\lim _{s \rightarrow+\infty} k(s)=0 .
$$

Notice that (2.7) and (2.8) imply that

$$
\begin{aligned}
& 0 \leq \mu(s) \leq \mu(0) \exp (-\delta s), \\
& 0 \leq k(s) \leq \frac{\mu(0)}{\delta} \exp (-\delta s), \quad \forall s \in \mathbb{R}_{+} .
\end{aligned}
$$

Let a function $u(x, t)$ be given such that $u \in L_{2}^{b}\left(\mathbb{R} ; H_{0}^{1}(\Omega)\right)$. The latter means that

$$
\|u\|_{L_{2}^{b}\left(\mathbb{R} ; H_{0}^{1}(\Omega)\right)}^{2}=\sup _{t \in \mathbb{R}} \int_{t}^{t+1}|\nabla u(s)|^{2} d s<\infty .
$$

As usual, we write $u(t)=u(x, t)$, omitting the argument $x$. We rewrite the memory term in (2.1) as follows:

$$
\begin{aligned}
\int_{0}^{\infty} k(s) \Delta u(t-s) d s & =\int_{0}^{\infty} k(s) \frac{d}{d s}\left(\int_{0}^{s} \Delta u(t-\tau) d \tau\right) d s \\
& =-\int_{0}^{\infty} k^{\prime}(s) \Delta\left(\int_{0}^{s} u(t-\tau) d \tau\right) d s \\
& =-\int_{0}^{\infty} k^{\prime}(s) \Delta \eta_{u}(t, s) d s .
\end{aligned}
$$

Here, integrating by parts in $s \in[0,+\infty)$, we have used condition (2.8), inequality (2.10), and assumption (2.11). Furthermore, we have set

$$
\eta_{u}(t, s) \equiv \eta_{u}(x, t, s)=\int_{0}^{s} u(x, t-\tau) d \tau=\int_{t-s}^{t} u(x, \tau) d \tau .
$$

All the calculations have a sense and are carried out in the space $H^{-1}(\Omega)$. Furthermore, all the integrals have a sense in the sense of Bochner. Notice that (2.13) implies that

$$
\begin{aligned}
& \partial_{s} \eta_{u}(t, s)=u(t-s), \\
& \partial_{t} \eta_{u}(t, s)=u(t)-u(t-s)=u(t)-\partial_{s} \eta_{u}(t, s) .
\end{aligned}
$$


Hence,

$$
u(t)=\partial_{t} \eta_{u}(t, s)+\partial_{s} \eta_{u}(t, s) .
$$

This identity will be extremely important in the sequel.

Using (2.12) and (2.6), we rewrite equation (2.1) as follows:

$$
\partial_{t} u(t)=\Delta u(t)+\int_{0}^{\infty} \mu(s) \Delta \eta_{u}(t, s) d s-f(u(t))+g .
$$

Following the general scheme of Section 1 , we assume that the function $u(x, t)$ is known for all $t \leq 0$. We write this formally as the initial condition for equation (2.16):

$$
\left.u\right|_{t \leq 0}=v(t), \quad t \leq 0 .
$$

We assume that the function $v(s), s \leq 0$, belongs to $L_{2}^{b}\left(\mathbb{R}_{-} ; H_{0}^{1}(\Omega)\right)$, that is,

$$
\|v\|_{L_{2}^{b}\left(\mathbb{R}_{-} ; H_{0}^{1}(\Omega)\right)}^{2}=\sup _{t \leq 0} \int_{t-1}^{t}|\nabla v(s)|^{2} d s<\infty .
$$

We shall specify the space $\mathcal{E}^{-}$of initial data later on.

We now clarify the notion of a solution for (2.1)-(2.2) (or, equivalently, for (2.16)-(2.2)). Let $u(\cdot)$ belong to $L_{2}^{\text {loc }}\left(\mathbb{R} ; H_{0}^{1}(\Omega)\right)$. Then, obviously, $\Delta u(\cdot) \in$ $L_{2}^{\text {loc }}\left(\mathbb{R} ; H^{-1}(\Omega)\right)$ and, due to $(2.9)$ and $(2.17)$, the function

$$
\psi(t)=\int_{0}^{\infty} \mu(s) \Delta \eta_{u}(t, s) d s \in L_{2}^{\text {loc }}\left(\mathbb{R} ; H^{-1}(\Omega)\right)
$$

as well. At the same time, if it is known that $u(\cdot)$ belongs to $L_{p}^{\text {loc }}\left(\mathbb{R}_{+} ; L_{p}(\Omega)\right)$, then, by $(2.5), f(u(\cdot)) \in L_{q}^{\text {loc }}\left(\mathbb{R}_{+} ; L_{q}(\Omega)\right)$, where $1 / p+1 / q=1$. Therefore, the right-hand side of equation (2.16) (or (2.1)) belongs to the space

$$
L_{2}^{\text {loc }}\left(\mathbb{R}_{+} ; H^{-1}(\Omega)\right)+L_{q}^{\text {loc }}\left(\mathbb{R}_{+} ; L_{q}(\Omega)\right) \subset L_{q}^{\text {loc }}\left(\mathbb{R}_{+} ; H^{-r}(\Omega)\right),
$$

where $r=\max \{1, n / 2-n / p\}$ and $\rho=n / 2-n / p$ is the exponent in the corresponding Sobolev embedding theorem (namely, $H_{0}^{\rho}(\Omega) \subset L_{p}(\Omega)$ and, by duality, $\left.L_{q}(\Omega) \subset H^{-\rho}(\Omega)\right)$. Hence, if $u \in L_{2}^{\text {loc }}\left(\mathbb{R} ; H_{0}^{1}(\Omega)\right) \cap L_{p}^{\text {loc }}\left(\mathbb{R}_{+} ; L_{p}(\Omega)\right)$, then equation (2.16), for $t \geq 0$, can be treated in the sense of distributions of the space $D^{\prime}\left(\mathbb{R}_{+} ; H^{-r}(\Omega)\right)$ (see [14]).

In order to give a sense to the initial condition (2.17) for $u(t)$, we assume that the functions $u(\cdot)$ and $v(\cdot)$ are continuous in time with values in $L_{2}(\Omega)$, that is, $v \in C\left(\mathbb{R}_{-} ; L_{2}(\Omega)\right)$ and $u \in C\left(\mathbb{R} ; L_{2}(\Omega)\right)$. 
Theorem 2.1. Let a function $v(\cdot)$ belong to the space $L_{2}^{b}\left(\mathbb{R}_{-} ; H_{0}^{1}(\Omega)\right) \cap$ $C\left(\mathbb{R}_{-} ; L_{2}(\Omega)\right)$. Then, under assumptions (2.3)-(2.8), problem (2.16), (2.2), and (2.17) (or (2.1), (2.2), and (2.17)) has a unique solution $u(x, t)$ such that

$$
u \in L_{\infty}\left(\mathbb{R}_{+} ; L_{2}(\Omega)\right) \cap L_{2}^{b}\left(\mathbb{R}_{+} ; H_{0}^{1}(\Omega)\right) \cap L_{p}^{\text {loc }}\left(\mathbb{R}_{+} ; L_{p}(\Omega)\right) \cap C\left(\mathbb{R}_{+} ; L_{2}(\Omega)\right) .
$$

Proof. A similar result is proved in [9]. The proof is analogous to that performed for the similar semilinear parabolic equation without memory, i.e., for $k \equiv 0$. We shall sketch here only the main steps (for more details, see $[1,2,14,19]$ ).

1. Faedo-Galerkin approximations. We consider the system of ordinary differential equations with memory of the form:

$$
\begin{aligned}
& \partial_{t} u_{N}(t)=\Delta u_{N}(t)+\int_{0}^{\infty} k(s) \Delta u_{N}(t-s) d s-P_{N} f\left(u_{N}(t)\right)+P_{N} g, \\
& \left.u_{N}\right|_{t \leq 0}=P_{N} v(t), \quad t \leq 0 .
\end{aligned}
$$

Here, $u_{N}(t)=u_{N}(x, t)=\sum_{j=1}^{N} c_{j N}(t) w_{j}(x)$, where $w_{j}(x), j=1, \ldots, N$, are the eigenfunctions of the operator $-\Delta$ of the Dirichlet problem:

$$
-\Delta w_{j}(x)=\lambda_{j} w_{j}(x),\left.\quad w_{j}\right|_{\partial \Omega}=0, \quad 0<\lambda_{1} \leq \lambda_{2} \leq \cdots \leq \lambda_{j} \rightarrow+\infty .
$$

Notice that $w_{j}(\cdot) \in H^{2}(\Omega) \cap H_{0}^{1}(\Omega) \cap L_{\infty}(\Omega)$ if, for instance, the boundary $\partial \Omega$ is a Lipschitz manifold. Furthermore, $P_{N}$ denotes the orthogonal projector in $L_{2}(\Omega)$ onto the subspace $H_{N}=\operatorname{span}\left\{w_{1}, w_{2}, \ldots, w_{N}\right\}$.

The system of ordinary differential equations (2.18) with initial datum (2.19) (with respect to the unknown scalar functions $c_{j N}(t), t \geq 0, j=1, \ldots, N$ ) has a solution $u_{N}(t)$ on a semi-interval $\left[0, T_{N}\right)$. (The proof uses the standard iteration method; the memory term does not cause any difficulty.) Moreover, $u_{N}(t) \in C^{1}\left(\left[0, T_{N}\right) ; H_{0}^{1}(\Omega) \cap L_{p}(\Omega)\right)$. We now prove that $T_{N}=+\infty$ by using the first a priori estimate for (2.18) (as well as for (2.1)).

2. The first a priori estimate and the existence of a solution. As for (2.1) and (2.16), we rewrite equation (2.18) as follows:

$$
\partial_{t} u_{N}(t)=\Delta u_{N}(t)+\int_{0}^{\infty} \mu(s) \Delta \eta_{u_{N}}(t, s) d s-P_{N} f\left(u_{N}(t)\right)+P_{N} g,
$$

where $\eta_{u_{N}}(t, s)=\int_{t-s}^{t} u_{N}(\tau) d \tau$ (see (2.13)). We now multiply equation (2.20) by the function $u_{N}(t)$ for any $t \in\left[0, T_{N}\right)$ and integrate this product over $\Omega$. After 
standard transformations, we obtain the equality

$$
\begin{aligned}
\frac{1}{2} \frac{d}{d t}\left|u_{N}(t)\right|^{2}+\left|\nabla u_{N}(t)\right|^{2}=\int_{0}^{\infty} & \mu(s)\left(\Delta \eta_{u_{N}}(t, s), u_{N}(t)\right) d s \\
& -\left(f\left(u_{N}(t)\right), u_{N}(t)\right)+\left(g, u_{N}(t)\right) .
\end{aligned}
$$

We shall omit the index $N$ and write $u(t) \stackrel{\text { def }}{\equiv} u_{N}(t)$. Here, we have also used the notations $|\cdot|$ and $(\cdot, \cdot)$ for the norm and the scalar product in $L_{2}(\Omega)$, respectively. We transform the integral term in (2.21), using identity (2.15), as follows:

$$
\begin{aligned}
\int_{0}^{\infty} \mu(s)\left(\Delta \eta_{u}(t, s), u(t)\right) d s \\
=\int_{0}^{\infty} \mu(s)\left(\Delta \eta_{u}(t, s), \partial_{t} \eta_{u}(t, s)+\partial_{s} \eta_{u}(t, s)\right) d s \\
=\int_{0}^{\infty} \mu(s)\left(\Delta \eta_{u}(t, s), \partial_{t} \eta_{u}(t, s)\right) d s \\
\quad+\int_{0}^{\infty} \mu(s)\left(\Delta \eta_{u}(t, s), \partial_{s} \eta_{u}(t, s)\right) d s .
\end{aligned}
$$

We note that

$$
\begin{aligned}
\int_{0}^{\infty} \mu & (s)\left(\Delta \eta_{u}(t, s), \partial_{t} \eta_{u}(t, s)\right) d s \\
= & -\frac{1}{2} \int_{0}^{\infty} \mu(s) \frac{d}{d t}\left|\nabla \eta_{u}(t, s)\right|^{2} d s \\
= & -\frac{1}{2} \frac{d}{d t} \int_{0}^{\infty} \mu(s)\left|\nabla \eta_{u}(t, s)\right|^{2} d s .
\end{aligned}
$$

Here, we have integrated by parts in $x \in \Omega$. All the transformations have a sense since the function $u(x, t)$ is smooth for $t \in\left[0, T_{N}\right)$. Concerning the second integral in the right-hand side of (2.22), we have

$$
\begin{aligned}
\int_{0}^{\infty} \mu & (s)\left(\Delta \eta_{u}(t, s), \partial_{s} \eta_{u}(t, s)\right) d s \\
= & -\frac{1}{2} \int_{0}^{\infty} \mu(s) \frac{d}{d s}\left|\nabla \eta_{u}(t, s)\right|^{2} d s \\
= & \frac{1}{2} \int_{0}^{\infty} \mu^{\prime}(s)\left|\nabla \eta_{u}(t, s)\right|^{2} d s .
\end{aligned}
$$

Here, we have first integrated by parts in $x \in \Omega$ and then in $s$, using the fact that $\mu(+\infty)=0$ (see (2.9)). Inserting (2.23) and (2.24) into (2.22) and then into 
(2.21), we obtain

$$
\begin{aligned}
\frac{1}{2} \frac{d}{d t}\{\mid & \left.\left.u(t)\right|^{2}+\int_{0}^{\infty} \mu(s)\left|\nabla \eta_{u}(t, s)\right|^{2} d s\right\}+|\nabla u(t)|^{2} \\
& -\frac{1}{2} \int_{0}^{\infty} \mu^{\prime}(s)\left|\nabla \eta_{u}(t, s)\right|^{2} d s=-(f(u(t)), u(t))+(g, u(t)) .
\end{aligned}
$$

Notice that $-\mu^{\prime}(s) \geq 0$ (see (2.6) and (2.7)) and, besides,

$$
-(f(u(t)), u(t)) \leq-\gamma \|\left. u(t)\right|_{L_{p}(\Omega)} ^{p}+C|\Omega|
$$

(see (2.4)). The Cauchy-Schwarz inequality implies

$$
\begin{aligned}
(g, u(t)) & \leq \frac{1}{2}|\nabla u(t)|^{2}+\frac{1}{2}\|g\|_{H^{-1}(\Omega)}^{2} \\
& \leq \frac{1}{2}|\nabla u(t)|^{2}+\frac{1}{2} \frac{|g|^{2}}{\lambda_{1}} .
\end{aligned}
$$

Recall that $\lambda_{1}$ is the first eigenvalue of the laplacian. Using (2.26) and (2.27) and integrating $(2.25)$ over $[0, t]$, we obtain

$$
\begin{aligned}
|u(t)|^{2}+ & \int_{0}^{\infty} \mu(s)\left|\nabla \eta_{u}(t, s)\right|^{2} d s+\int_{0}^{t}|\nabla u(\tau)|^{2} d \tau+2 \gamma \int_{0}^{t}\|u(\tau)\|_{L_{p}(\Omega)}^{p} d \tau \\
& \leq|u(0)|^{2}+\int_{0}^{\infty} \mu(s)\left|\nabla \eta_{u}(0, s)\right|^{2} d s+C_{0} t \\
& \leq|v(0)|^{2}+\int_{0}^{\infty} \mu(s)\left|\nabla \eta_{v}(0, s)\right|^{2} d s+C_{0} t
\end{aligned}
$$

where $C_{0}=2 C|\Omega|+|g|^{2} / \lambda_{1}$. Recall that $u(\cdot)=u_{N}(\cdot)$. We note that the right-hand side of (2.29) is independent of $N$, since $v \in L_{2}^{b}\left(\mathbb{R}_{-} ; H_{0}^{1}(\Omega)\right)$ and $\mu$ satisfies (2.9). Hence, we may assume that $T_{N}=+\infty$. Moreover, the sequence $\left\{u_{N}(t)\right\}$ is bounded in the spaces $L_{\infty}\left(\mathbb{R}_{+} ; L_{2}(\Omega)\right), L_{2}^{b}\left(\mathbb{R}_{+} ; H_{0}^{1}(\Omega)\right)$, and $L_{p}^{\text {loc }}\left(\mathbb{R}_{+} ; L_{p}(\Omega)\right)$. Therefore, it follows from equation (2.18) that the sequence $\left\{\partial_{t} u_{N}(t)\right\}$ is bounded in $L_{q}^{\text {loc }}\left(\mathbb{R}_{+} ; H^{-r}(\Omega)\right)$. Consequently, we may pass to the limit in equations (2.18) and (2.19) as $N_{k} \rightarrow \infty$, for a proper subsequence $N_{k}$, and obtain a function $u \in L_{\infty}\left(\mathbb{R}_{+} ; L_{2}(\Omega)\right) \cap L_{2}^{b}\left(\mathbb{R}_{+} ; H_{0}^{1}(\Omega)\right) \cap L_{p}^{\text {loc }}\left(\mathbb{R}_{+} ; L_{p}(\Omega)\right)$ which satisfies $(2.16)$ in the space $D^{\prime}\left(\mathbb{R}_{+} ; H^{-r}(\Omega)\right)$. This function also satisfies the initial condition (2.17).

For the moment, we can state that the solution $u$ belongs to $C\left(\mathbb{R}_{+} ; H^{-r}(\Omega)\right)$. The following lemma implies that $u \in C_{w}\left(\mathbb{R}_{+} ; L_{2}(\Omega)\right)$, where $C_{w}\left(\mathbb{R}_{+} ; X\right)$ denotes the space of weakly continuous functions with values in a Banach space $X$.

Lemma 2.2. Let $X$ and $Y$ be two Banach spaces such that $X \subset Y$. If a function $\varphi \in L^{\infty}\left(\mathbb{R}_{+} ; X\right)$ and $\varphi \in C_{w}\left(\mathbb{R}_{+} ; Y\right)$, then $\varphi \in C_{w}\left(\mathbb{R}_{+} ; X\right)$.

See $[15,20]$ for the proof. Later on, we shall prove that $u(\cdot)$ actually belongs to $C\left(\mathbb{R} ; L_{2}(\Omega)\right)$. 
3. Uniqueness of the solution. We use the following result.

Lemma 2.3. Let $H$ be a Hilbert space. Let also $V, E$, and $X$ be Banach spaces such that $V \subseteq H \subseteq V^{\prime} \subseteq X$ and $E \subseteq H \subseteq E^{\prime} \subseteq X$, where the spaces $V^{\prime}$ and $E^{\prime}$ are the duals of $V$ and $E$, respectively. Here, the space $H^{\prime}$ is identified with $H$. Assume that $u(s) \in L_{2}(0, M ; V) \cap L_{p}(0, M ; E)$ (for a fixed $\left.p>1\right)$ and that the distribution $\partial_{t} u(s)$ from $D^{\prime}(0, M ; X)$ can be represented in the form $\partial_{t} u(s)=w(s)+h(s)$, where $w \in L_{2}\left(0, M ; V^{\prime}\right)$ and $h \in L_{q}\left(0, M ; E^{\prime}\right)(1 / p+1 / q=1)$. Then

(i) the function $\|u(s)\|_{H}^{2}$ is absolutely continuous on $[0, M]$. Moreover,

$$
\begin{aligned}
\frac{d}{d t}\|u(t)\|_{H}^{2} & =2\left\langle\partial_{t} u(t), u(t)\right\rangle \\
& =2\langle w(t), u(t)\rangle+2\langle h(t), u(t)\rangle ;
\end{aligned}
$$

(ii) $u(\cdot) \in C([0, M] ; H)$.

This statement is a generalization of known interpolation results. See [15] and [20, Chapter 3, Lemma 1.2], where the case $V=E, p=2$, and $V$ is a Hilbert space is considered.

Let two solutions $u_{1}(t)$ and $u_{2}(t)$ of $(2.1)-(2.2)$ with the same initial datum (2.17) be given. We set $w(t)=u_{1}(t)-u_{2}(t)$. It is clear that $w \in$ $L_{2}^{b}\left(\mathbb{R}_{+} ; H_{0}^{1}(\Omega)\right) \cap L_{p}^{\text {loc }}\left(\mathbb{R}_{+} ; L_{p}(\Omega)\right), w \in C_{w}\left(\mathbb{R}_{+} ; L_{2}(\Omega)\right)$, and $w$ satisfies the equations

$$
\begin{aligned}
& \partial_{t} w(t)=\Delta w(t)+\int_{0}^{\infty} \mu(s) \Delta \eta_{w}(t, s) d s-\left(f\left(u_{1}(t)\right)-f\left(u_{2}(t)\right)\right), \\
& \left.w\right|_{\partial \Omega}=0,\left.\quad w\right|_{t \leq 0}=0
\end{aligned}
$$

where

$$
\eta_{w}(t, s)=\int_{t-s}^{t} w(\tau) d \tau=\int_{t-s}^{t}\left(u_{1}(\tau)-u_{2}(\tau)\right) d \tau .
$$

From (2.31), it follows that

$$
\partial_{t} w \in L_{2}^{\text {loc }}\left(\mathbb{R}_{+} ; H^{-1}(\Omega)\right)+L_{q}^{\text {loc }}\left(\mathbb{R}_{+} ; L_{q}(\Omega)\right) \subset L_{q}^{\text {loc }}\left(\mathbb{R}_{+} ; H^{-r}(\Omega)\right) .
$$

Therefore, Lemma 2.3 is applicable with $H=L_{2}(\Omega), V=H_{0}^{1}(\Omega), E=L_{p}(\Omega)$, $V^{\prime}=H^{-1}(\Omega), E^{\prime}=L_{q}(\Omega), X=H^{-r}(\Omega)$ and, for equation (2.31), identity (2.30) takes the form

$$
\begin{aligned}
& \frac{1}{2} \frac{d}{d t}|w(t)|^{2}+|\nabla w(t)|^{2} \\
& \quad=\int_{0}^{\infty} \mu(s)\left(\Delta \eta_{w}(t, s), w(t)\right) d s-\left(f\left(u_{1}(t)\right)-f\left(u_{2}(t)\right), w(t)\right)
\end{aligned}
$$


We note that, due to (2.15), $w(t)=\partial_{t} \eta_{w}(t, s)+\partial_{t} \eta_{w}(t, s)$, and we have the identity

$$
\begin{aligned}
& \int_{0}^{\infty} \mu(s)\left(\Delta \eta_{w}(t, s), w(t)\right) d s \\
& \quad=-\frac{1}{2} \frac{d}{d t} \int_{0}^{\infty} \mu(s)\left|\nabla \eta_{w}(t, s)\right|^{2} d s+\frac{1}{2} \int_{0}^{\infty} \mu^{\prime}(s)\left|\nabla \eta_{w}(t, s)\right|^{2} d s .
\end{aligned}
$$

(Compare with (2.22)-(2.24).) To prove (2.33), we apply Lemma 2.3. Using (2.3), we obtain the inequality

$$
-\left(f\left(u_{1}(t)\right)-f\left(u_{2}(t)\right), w(t)\right) \leq D|w(t)|^{2} .
$$

Inserting (2.33) into (2.32), we deduce that

$$
\begin{aligned}
\frac{1}{2} \frac{d}{d t}\left\{|w(t)|^{2}+\int_{0}^{\infty} \mu(s)\left|\nabla \eta_{w}(t, s)\right|^{2} d s\right\} & +|\nabla w(t)|^{2} \\
& -\frac{1}{2} \int_{0}^{\infty} \mu^{\prime}(s)\left|\nabla \eta_{w}(t, s)\right|^{2} d s \leq D|w(t)|^{2} .
\end{aligned}
$$

Recall that $\mu^{\prime}(s) \leq 0$ for all $s \geq 0$ (see (2.6) and (2.7)). Therefore,

$$
\frac{1}{2} \frac{d}{d t}\left\{|w(t)|^{2}+\int_{0}^{\infty} \mu(s)\left|\nabla \eta_{w}(t, s)\right|^{2} d s\right\} \leq D|w(t)|^{2} .
$$

Hence, integrating (2.34) over $[0, t]$, we have

$$
\begin{aligned}
& |w(t)|^{2}+\int_{0}^{\infty} \mu(s)\left|\nabla \eta_{w}(t, s)\right|^{2} d s \\
& \quad \leq|w(0)|^{2}+\int_{0}^{\infty} \mu(s)\left|\nabla \eta_{w}(0, s)\right|^{2} d s+2 D \int_{0}^{t}|w(\tau)|^{2} d \tau
\end{aligned}
$$

Finally, since $\mu(s) \geq 0$ for all $s \geq 0$, we obtain the inequality

(2.36) $|w(t)|^{2} \leq|w(0)|^{2}+\int_{0}^{\infty} \mu(s)\left|\nabla \eta_{w}(0, s)\right|^{2} d s+2 D \int_{0}^{t}|w(\tau)|^{2} d \tau$.

Since $w(t)=0$ for $t \leq 0$, we deduce that $|w(0)|=0$ and

$$
\eta_{w}(0, s)=\int_{0-s}^{0} w(\tau) d \tau=0, \quad \forall s \geq 0 .
$$

Hence, inequality (2.36) yields

$$
|w(t)|^{2} \leq 2 D \int_{0}^{t}|w(\tau)|^{2} d \tau, \quad \forall t \geq 0 .
$$


Consequently, using the Gronwall lemma, we conclude that $w(t)=0$ for all $t \geq 0$, i.e., $u_{1}(t)=u_{2}(t)$ for all $t \geq 0$.

Notice that Lemma 2.3 also implies that $u \in C\left(\mathbb{R}_{+} ; L_{2}(\Omega)\right)$.

\section{The Main Estimates for the Solutions of THE HEAT EQUATION WITH MEMORY}

We now prove some inequalities for the solutions of (2.1)-(2.2). We shall use these estimates to construct trajectory and global attractors for $(2.1)-(2.2)$ in the next section.

Proposition 3.1. Let $u(t), t \geq 0$, be any solution of problem (2.1), (2.2), and (2.17),

$$
u \in C\left(\mathbb{R}_{+} ; L_{2}(\Omega)\right) \cap L_{2}^{b}\left(\mathbb{R}_{+} ; H_{0}^{1}(\Omega)\right) \cap L_{p}^{\text {loc }}\left(\mathbb{R}_{+} ; L_{p}(\Omega)\right) .
$$

Then, the following inequalities hold:

$$
\varphi(t) \leq \varphi(0) e^{-\varkappa t}+\frac{C_{0}}{\varkappa}, \quad \forall t \geq 0,
$$

where $x=\min \left\{\lambda_{1}, \delta\right\}, C_{0}=2 C|\Omega|+|g|^{2} \lambda_{1}^{-1}$, and

$$
\varphi(t)=|u(t)|^{2}+\int_{0}^{\infty} \mu(s)\left|\nabla \eta_{u}(t, s)\right|^{2} d s .
$$

Moreover,

$$
\begin{aligned}
\varphi(t)+\int_{\tau}^{t}|\nabla u(s)|^{2} d s+2 \gamma \int_{\tau}^{t}\|u(s)\|_{L_{p}(\Omega)}^{p} d s \leq \varphi(\tau)+C_{0}(t-\tau), \\
\forall t \geq \tau \geq 0 ; \\
\int_{0}^{t} k(t-s)|\nabla u(s)|^{2} d s=\int_{0}^{t} k(s)|\nabla u(t-s)|^{2} d s \\
\leq \varphi(0) \mu(0)\left(\frac{e^{-\delta t}}{\delta}+t e^{-\varkappa t}\right)+\frac{C_{0}}{\varkappa} k(0)+C_{0} \alpha, \\
\forall t \geq 0,
\end{aligned}
$$

where $\alpha=\int_{0}^{\infty} k(s) d s$.

Proof. This can be done in two ways. First, we can prove these inequalities for the Faedo-Galerkin approximations, i.e., for a solution of (2.18), and then we can pass to the limit in the inequalities that we obtain. The second technique is based on Lemma 2.3 which legitimates all the transformations below. 
Similarly to (2.25), we prove the identity

$$
\begin{aligned}
\frac{1}{2} \frac{d}{d t}\left\{|u(t)|^{2}+\int_{0}^{\infty} \mu(s)\left|\nabla \eta_{u}(t, s)\right|^{2} d s\right\} & +|\nabla u(t)|^{2} \\
& -\frac{1}{2} \int_{0}^{\infty} \mu^{\prime}(s)\left|\nabla \eta_{u}(t, s)\right|^{2} d s=-(f(u(t)), u(t))+(g, u(t)) .
\end{aligned}
$$

We now apply inequalities (2.26), (2.27), and (2.7) and obtain

(3.5) $\frac{d}{d t} \varphi(t)+|\nabla u(t)|^{2}+2 \gamma\|u(t)\|_{L_{p}(\Omega)}^{p}+\delta \int_{0}^{\infty} \mu(s)\left|\nabla \eta_{u}(t, s)\right|^{2} d s \leq C_{0}$, where $C_{0}=2 C|\Omega|+|g|^{2} / \lambda_{1}$. Using the Poincaré inequality $|\nabla u|^{2} \geq \lambda_{1}|u|^{2}$, we conclude from (3.5) that

$$
\frac{d}{d t} \varphi(t)+\varkappa \varphi(t)+2 \gamma\|u(t)\|_{L_{p}(\Omega)}^{p} \leq C_{0} \quad\left(\varkappa=\min \left\{\lambda_{1}, \delta\right\}\right) .
$$

This implies, in a standard way,

$$
\varphi(t) \leq \varphi(0) e^{-\varkappa t}+\frac{C_{0}}{\varkappa} .
$$

Integrating (3.5) in $t$, we obtain

$$
\begin{array}{r}
\varphi(t)+\int_{\tau}^{t}|\nabla u(s)|^{2} d s+2 \gamma \int_{\tau}^{t}\|u(s)\|_{L_{p}(\Omega)}^{p} d s \leq \varphi(\tau)+C_{0}(t-\tau), \\
\forall t \geq \tau \geq 0 .
\end{array}
$$

Inequalities (3.1) and (3.2) are proved. We now prove (3.3). It follows from (3.5) that

$$
\varphi^{\prime}(s)+|\nabla u(s)|^{2} \leq C_{0}, \quad \forall s \geq 0 .
$$

We then multiply (3.9) by $k(t-s)$ and integrate over $[0, t]$ in $s$ :

$$
\begin{aligned}
\int_{0}^{t} \varphi^{\prime}(s) k(t-s) d s+ & \int_{0}^{t} k(t-s)|\nabla u(s)|^{2} d s \\
& \leq C_{0} \int_{0}^{t} k(t-s) d s \leq C_{0} \int_{0}^{\infty} k(s) d s=C_{0} \alpha,
\end{aligned}
$$

where $\alpha=\int_{0}^{\infty} k(s) d s$. We integrate by parts in the first integral in the left-hand side of (3.10):

$$
\begin{aligned}
\int_{0}^{t} \varphi^{\prime}(s) k(t-s) d s & =\varphi(t) k(0)-\varphi(0) k(t)+\int_{0}^{t} k^{\prime}(t-s) \varphi(s) d s \\
& \geq-\varphi(0) k(t)-\int_{0}^{t} \mu(t-s) \varphi(s) d s .
\end{aligned}
$$


From (3.10), (3.11), and (2.10), we obtain

$$
\begin{aligned}
\int_{0}^{t} k(t-s)|\nabla u(s)|^{2} d s & \leq \varphi(0) k(t)+\int_{0}^{t} \mu(t-s) \varphi(s) d s+C_{0} \alpha \\
& \leq \varphi(0) \mu(0) \frac{e^{-\delta t}}{\delta}+\int_{0}^{t} \mu(t-s) \varphi(s) d s+C_{0} \alpha
\end{aligned}
$$

It follows from (2.9) that

$$
\mu(t-s) \leq \mu(0) e^{-\delta(t-s)} \leq \mu(0) e^{-\varkappa(t-s)} \text { for } t-s \geq 0,
$$

since $\delta \geq \varkappa$. Hence, (3.7) implies

$$
\begin{aligned}
\int_{0}^{t} \mu(t-s) \varphi(s) d s & \leq \varphi(0) \mu(0) \int_{0}^{t} e^{-\varkappa(t-s)} e^{-\varkappa s} d s+\frac{C_{0}}{\varkappa} \int_{0}^{t} \mu(t-s) d s \\
& \leq \varphi(0) \mu(0) e^{-\varkappa t} t+\frac{C_{0}}{\varkappa} \int_{0}^{\infty} \mu(s) d s \\
& =\varphi(0) \mu(0) e^{-\varkappa t} t+\frac{C_{0}}{\varkappa} k(0) .
\end{aligned}
$$

Finally, estimate (3.12) implies

$$
\int_{0}^{t} k(t-s)|\nabla u(s)|^{2} d s \leq \varphi(0) \mu(0)\left(e^{-\varkappa t} t+\frac{e^{-\delta t}}{\delta}\right)+\frac{C_{0}}{\varkappa} k(0)+C_{0} \alpha .
$$

This finishes the proof.

We assume that the initial datum $v(\cdot)$ satisfies the inequality

$$
\varphi(0)=|v(0)|^{2}+\int_{0}^{\infty} \mu(s)\left|\nabla \eta_{v}(0, s)\right|^{2} d s \leq R^{2},
$$

where $R$ is an arbitrary positive number.

We obtain from (3.1) and (3.2) the following result.

Corollary 3.2. If the function $v(\cdot)$ satisfies (3.13), then the solution $u(\cdot)$ of problem (2.1), (2.2), and (2.17) satisfies the following inequalities:

$$
\begin{aligned}
& \varphi(t)=|u(t)|^{2}+\int_{0}^{\infty} \mu(s)\left|\nabla \eta_{u}(t, s)\right|^{2} d s \leq R^{2} e^{-\varkappa t}+\frac{C_{0}}{\varkappa}, \\
& \int_{t}^{t+1}|\nabla u(s)|^{2} d s \leq R^{2} e^{-\varkappa t}+C_{0}^{\prime}, \\
& \int_{0}^{t}|\nabla u(s)|^{2} d s+2 \gamma \int_{0}^{t}\|u(s)\|_{L_{p}(\Omega)}^{p} d s \leq R^{2}+C_{0} t, \quad \forall t \geq 0,
\end{aligned}
$$

where $C_{0}^{\prime}=C_{0} / x+C_{0}$. 
We now assume that the initial datum $v(\cdot)$ satisfies the conditions

$$
\begin{gathered}
\varphi(0) \leq R^{2}, \\
\int_{0}^{\infty} e^{-\varkappa s}|\nabla v(-s)|^{2} d s \leq R^{2} .
\end{gathered}
$$

Corollary 3.3. If the function $v(\cdot)$ satisfies (3.17), then the corresponding solution $u(\cdot)$ satisfies the inequalities:

$$
\begin{aligned}
& \int_{0}^{\infty} k(s)|\nabla u(t-s)|^{2} d s \leq c_{1} R^{2}(1+t) e^{-\varkappa t}+C_{1}, \\
& \int_{0}^{\infty} \mu(s)|\nabla u(t-s)|^{2} d s \leq c_{2} R^{2}(1+t) e^{-\varkappa t}+C_{2}, \\
& \int_{0}^{\infty} e^{-\varkappa s}|\nabla u(t-s)|^{2} d s \leq c_{3} R^{2}(1+t) e^{-\varkappa t}+C_{3}, \quad \forall t \geq 0 .
\end{aligned}
$$

Proof. We have

$$
\begin{aligned}
\int_{0}^{\infty} k(s)|\nabla u(t-s)|^{2} d s=\int_{0}^{t} k(s)|\nabla u(t-s)|^{2} d s & \\
& +\int_{t}^{\infty} k(s)|\nabla u(t-s)|^{2} d s .
\end{aligned}
$$

We estimate the second integral in the right-hand side of (3.21) by using (2.10):

$$
\begin{aligned}
\int_{t}^{\infty} k(s)|\nabla u(t-s)|^{2} d s & \leq \frac{\mu(0)}{\delta} \int_{t}^{\infty} e^{-\delta s}|\nabla u(t-s)|^{2} d s \\
& \leq \frac{\mu(0)}{\delta} \int_{t}^{\infty} e^{-\varkappa s}|\nabla u(t-s)|^{2} d s \\
& =\frac{\mu(0)}{\delta} e^{-\varkappa t} \int_{0}^{\infty} e^{-\varkappa s}|\nabla u(-s)|^{2} d s \\
& \leq \frac{\mu(0)}{\delta} e^{-\varkappa t} R^{2}=c_{3}^{\prime} e^{-\varkappa t} R^{2} .
\end{aligned}
$$

This estimate, together with (3.3) and (3.17), gives inequality (3.18) for appropriate constants $c_{3}$ and $C_{3}$, since $\mathcal{X} \leq \delta$.

To prove inequality (3.19) for the kernel $\mu(s)$, we use estimates (2.9) and (3.15). We have

$$
\begin{aligned}
\int_{0}^{\infty} \mu(s)|\nabla u(t-s)|^{2} d s=\int_{0}^{t} \mu(s)|\nabla u(t-s)|^{2} d s & \\
& +\int_{t}^{\infty} \mu(s)|\nabla u(t-s)|^{2} d s .
\end{aligned}
$$


It follows from (2.9) that

$$
\mu(s) \leq \mu(0) e^{-\delta s} \leq \mu(0) e^{-\varkappa s}, \quad \forall s \geq 0 .
$$

Concerning the second integral in the right-hand side of (3.23), we obtain, similarly to (3.22),

$$
\begin{aligned}
\int_{t}^{\infty} \mu(s)|\nabla u(t-s)|^{2} d s & \leq \mu(0) \int_{t}^{\infty} e^{-\delta s}|\nabla u(t-s)|^{2} d s \\
& \leq \mu(0) e^{-\varkappa t} R^{2}=c_{2}^{\prime \prime} R^{2} e^{-\varkappa t} .
\end{aligned}
$$

We now estimate the first integral in the right-hand side of (3.23). We have

$$
\begin{aligned}
& \int_{0}^{t} \mu(s)|\nabla u(t-s)|^{2} d s \\
= & \int_{0}^{t} \mu(t-s)|\nabla u(s)|^{2} d s \\
= & \int_{t-1}^{t} \mu(t-s)|\nabla u(s)|^{2} d s+\int_{t-2}^{t-1} \mu(t-s)|\nabla u(s)|^{2} d s \\
& \quad+\cdots+\int_{0}^{t-[t]} \mu(t-s)|\nabla u(s)|^{2} d s \\
\leq & \mu(0) \int_{t-1}^{t}|\nabla u(s)|^{2} d s+\mu(0) e^{-\varkappa} \int_{t-2}^{t-1}|\nabla u(s)|^{2} d s \\
& \quad+\cdots+\mu(0) e^{-\varkappa[t]} \int_{0}^{t-[t]}|\nabla u(s)|^{2} d s \\
\leq & \mu(0)\left(R^{2} e^{-\varkappa(t-1)}+C_{0}^{\prime}\right)+\mu(0) e^{-\varkappa}\left(R^{2} e^{-\varkappa(t-2)}+C_{0}^{\prime}\right) \\
& \quad+\cdots+\mu(0) e^{-\varkappa[t]}\left(R^{2} e^{-\varkappa(t-[t]-1)}+C_{0}^{\prime}\right) \\
= & \mu(0) R^{2} e^{\varkappa} e^{-\varkappa t}(\underbrace{1+1+\cdots+1}_{[t]+1 \text { times }})+\mu(0) C_{0}^{\prime}\left(1+e^{-\varkappa}+\cdots+e^{-[t] \varkappa}\right) \\
\leq & \mu(0) R^{2} e^{\varkappa} e^{-\varkappa t}(t+1)+\frac{\mu(0) C_{0}^{\prime}}{1-e^{-\varkappa}}=c_{2}^{\prime} R^{2} e^{-\varkappa t}(t+1)+C_{2} .
\end{aligned}
$$

Here, we have also used estimate (3.15). Combining then (3.24) and (3.25), we obtain (3.19). The proof of (3.20) simply repeats the reasoning above with the function $e^{-\varkappa s}$ in place of $\mu(s)$.

Corollary 3.4. Under the assumptions of Corollary 3.3, we have

$$
\begin{aligned}
& \int_{0}^{t} s|\nabla u(s)|^{2} d s \leq c_{4} R^{2}+C_{4}\left(t+t^{2}\right), \\
& \int_{0}^{t} \tau \int_{0}^{\infty} \mu(s)|\nabla u(\tau-s)|^{2} d s d \tau \leq c_{5} R^{2}+C_{5} t^{2}, \quad \forall t \geq 0 .
\end{aligned}
$$


Proof. To prove (3.26), we multiply inequality (3.9) by $s$ and integrate the resulting inequality over $[0, t]$ :

$$
\begin{aligned}
(s \varphi(s))^{\prime}+s|\nabla u(s)|^{2} & \leq \varphi(s)+s C_{0}, \quad \forall s \geq 0 \\
t \varphi(t)+\int_{0}^{t} s|\nabla u(s)|^{2} d s & \leq \int_{0}^{t} \varphi(s) d s+\frac{C_{0}}{2} t^{2} \\
& \leq \int_{0}^{t} \varphi(0) e^{-\varkappa s} d s+\int_{0}^{t} \frac{C_{0}}{\varkappa} d s+\frac{C_{0}}{2} t^{2} \\
& =\varphi(0) \frac{1-e^{-\varkappa s}}{\varkappa}+\frac{C_{0}}{\varkappa} t+\frac{C_{0}}{2} t^{2} \\
& \leq C_{4} R^{2}+C_{4}\left(t+t^{2}\right) .
\end{aligned}
$$

Here, we have used inequalities (3.1) and (3.13).

In order to prove (3.27), we multiply inequality (3.19) by $t$ and integrate over $[0, t]$.

We need one more important a priori estimate on the solutions of problem (2.1), (2.2), and (2.17).

Proposition 3.5. Let $v(\cdot)$ satisfy (3.17). Then, the corresponding solution $u(\cdot)$ satisfies the inequality

$$
\int_{0}^{t} \tau\left|\partial_{t} u(\tau)\right|^{2} d \tau+t|\nabla u(t)|^{2}+t|| u(t) \|_{L_{p}(\Omega)}^{p} \leq c_{6} R^{2}+C_{6} t^{2}, \quad \forall t>0,
$$

where the constants $c_{6}$ and $C_{6}$ are independent of $R$ and $t$.

Proof. We shall perform all the necessary transformations for the FaedoGalerkin approximation $u_{N}(t)$ of order $N$ and obtain (3.28) for $u_{N}(t)$. Then, we shall pass to the limit in this inequality as $N \rightarrow \infty$ and derive the same inequality for the exact solution. We shall omit the index $N$ for brevity.

Consider equation (2.1). We set

$$
\theta_{u}(t, s)=u(t)-u(t-s),
$$

and rewrite equation (2.1) as follows:

(3.30) $\partial_{t} u(t)=(1+\alpha) \Delta u(t)-\int_{0}^{\infty} k(s) \Delta \theta_{u}(t, s) d s-f(u(t))+g, \quad t \geq 0$, where $\alpha=\int_{0}^{\infty} k(s) d s$. It follows from (3.29) that

$$
\partial_{t} u(t)=\partial_{t} \theta_{u}(t, s)+\partial_{s} \theta_{u}(t, s) .
$$


We now take the scalar product in $L_{2}(\Omega)$ of equation (3.30) with $\partial_{t} u$ and obtain, after standard transformations, the following equality:

(3.32) $\left|\partial_{t} u(t)\right|^{2}+\frac{1}{2} \frac{d}{d t}\left\{(1+\alpha)|\nabla u(t)|^{2}+2 \int_{\Omega} F(u(x, t)) d x-2(g, u(t))\right\}$ $=-\int_{0}^{\infty} k(s)\left(\Delta \theta_{u}(t, s), \partial_{t} u(t)\right) d s, \quad t \geq 0$,

where $F(u)=\int_{0}^{u} f(w) d w$. Consider the integral term in the right-hand side of (3.32). Using (3.31), we have

$$
\begin{aligned}
\int_{0}^{\infty} & k(s)\left(\Delta \theta_{u}(t, s), \partial_{t} u(t)\right) d s \\
\quad= & \int_{0}^{\infty} k(s)\left(\Delta \theta_{u}(t, s), \partial_{t} \theta_{u}(t, s)+\partial_{s} \theta_{u}(t, s)\right) d s \\
= & \int_{0}^{\infty} k(s)\left(\Delta \theta_{u}, \partial_{t} \theta_{u}\right) d s+\int_{0}^{\infty} k(s)\left(\Delta \theta_{u}, \partial_{s} \theta_{u}\right) d s \\
= & -\frac{1}{2} \frac{d}{d t} \int_{0}^{\infty} k(s)\left|\nabla \theta_{u}(t, s)\right|^{2} d s-\frac{1}{2} \int_{0}^{\infty} k(s) \frac{d}{d s}\left|\nabla \theta_{u}(t, s)\right|^{2} d s .
\end{aligned}
$$

Here, we have integrated by parts in $x$ (recall that $\left.\left.\theta_{u}\right|_{\partial \Omega}=0\right)$ and have used the elementary identity $2 z(d z / d y)=d z^{2} / d y$.

In the second integral in (3.33), we integrate by parts in $s$. Recall that $k(+\infty)=0($ see $(2.8))$ and $\theta_{u}(t, 0)=u(t)-u(t)=0$. We thus rewrite (3.33) as

$$
\begin{aligned}
& \int_{0}^{\infty} k(s)\left(\Delta \theta_{u}(t, s), \partial_{t} u(t)\right) d s \\
&=-\frac{1}{2} \frac{d}{d t} \int_{0}^{\infty} k(s)\left|\nabla \theta_{u}(t, s)\right|^{2} d s+\frac{1}{2} \int_{0}^{\infty} k^{\prime}(s)\left|\nabla \theta_{u}(t, s)\right|^{2} d s .
\end{aligned}
$$

Replacing (3.34) into (3.32), we obtain

$$
\begin{aligned}
\left|\partial_{t} u(t)\right|^{2}+\frac{1}{2} & \frac{d}{d t}\left\{(1+\alpha)|\nabla u(t)|^{2}+2 \int_{\Omega} F(u(x, t)) d x-2(g, u(t))\right. \\
& \left.-\int_{0}^{\infty} k(s)\left|\nabla \theta_{u}(t, s)\right|^{2} d s\right\}=\frac{1}{2} \int_{0}^{\infty} \mu(s)\left|\nabla \theta_{u}(t, s)\right|^{2} d s,
\end{aligned}
$$

since $\mu(s)=-k^{\prime}(s)$. 
We now multiply (3.35) by $t$ and integrate the resulting equality over $[0, t]$. We have

$$
\begin{gathered}
\int_{0}^{t} \tau\left|\partial_{t} u(\tau)\right|^{2} d \tau+\frac{t}{2}\left\{(1+\alpha)|\nabla u(t)|^{2}+2 \int_{\Omega} F(u(x, t)) d x\right. \\
\left.-2(g, u(t))-\int_{0}^{\infty} k(s)\left|\nabla \theta_{u}(t, s)\right|^{2} d s\right\} \\
=\frac{1+\alpha}{2} \int_{0}^{t}|\nabla u(\tau)|^{2} d \tau+\int_{0}^{t} \int_{\Omega} F(u(x, \tau)) d x d \tau \\
-\int_{0}^{t}(g, u(\tau)) d \tau-\frac{1}{2} \int_{0}^{t} \int_{0}^{\infty} k(s)\left|\nabla \theta_{u}(t, s)\right|^{2} d s d t \\
+\frac{1}{2} \int_{0}^{t} \tau \int_{0}^{\infty} \mu(s)\left|\nabla \theta_{u}(\tau, s)\right|^{2} d s d \tau .
\end{gathered}
$$

Notice that, in (3.36), $k(s) \geq 0$ for all $s \geq 0$. Therefore,

$$
\begin{gathered}
\int_{0}^{t} \tau\left|\partial_{t} u(\tau)\right|^{2} d \tau+\frac{t}{2}\left\{(1+\alpha)|\nabla u(t)|^{2}+2 \int_{\Omega} F(u(x, t)) d x\right. \\
\left.-2(g, u(t))-\int_{0}^{\infty} k(s)\left|\nabla \theta_{u}(t, s)\right|^{2} d s\right\} \\
\leq \frac{1+\alpha}{2} \int_{0}^{t}|\nabla u(\tau)|^{2} d \tau \\
+\int_{0}^{t} \int_{\Omega} F(u(x, \tau)) d x d \tau-\int_{0}^{t}(g, u(\tau)) d \tau \\
+\frac{1}{2} \int_{0}^{t} \tau \int_{0}^{\infty} \mu(s)\left|\nabla \theta_{u}(\tau, s)\right|^{2} d s d \tau, \quad \forall t \geq 0 .
\end{gathered}
$$

It follows from (2.4) and (2.5) that

$$
\begin{aligned}
& \int_{\Omega} F(u(x, t)) d x \geq \gamma_{1}\|u(t)\|_{L_{p}(\Omega)}^{p}-C^{\prime}, \\
& \left|\int_{\Omega} F(u(x, t)) d x\right| \leq \gamma_{2}\left(\|u(t)\|_{L_{p}(\Omega)}^{p}+1\right) .
\end{aligned}
$$

Besides, we clearly have

$$
(g, u) \leq|g| \cdot|u| \leq|g| \cdot \frac{1}{\lambda_{1}^{1 / 2}}|\nabla u| \leq C_{\varepsilon}|g|^{2}+\varepsilon|\nabla u|^{2}, \quad \forall \varepsilon>0
$$


where $C_{\varepsilon}=1 /\left(4 \lambda_{1} \varepsilon\right)$. We apply (3.38), (3.39), and (3.40) in (3.37) and obtain

$$
\begin{aligned}
& \int_{0}^{t} \tau\left|\partial_{t} u(\tau)\right|^{2} d \tau \\
& \quad+\frac{t}{2}\left\{(1+\alpha-\varepsilon)|\nabla u(t)|^{2}+2 \gamma_{1}|| u(t) \|_{L_{p}(\Omega)}^{p}-\int_{0}^{\infty} k(s)\left|\nabla \theta_{u}(t, s)\right|^{2} d s\right\} \\
& \leq \gamma_{3} \int_{0}^{t}|\nabla u(\tau)|^{2} d \tau+\gamma_{2} \int_{0}^{t}\|u(\tau)\|_{L_{p}(\Omega)}^{p} d \tau \\
& \quad+\frac{1}{2} \int_{0}^{t} \tau \int_{0}^{\infty} \mu(s)\left|\nabla \theta_{u}(\tau, s)\right|^{2} d s d \tau+C_{\varepsilon}^{\prime} t, \quad \forall t \geq 0 .
\end{aligned}
$$

Here, the constants $\gamma_{1}, \gamma_{2}, \gamma_{3}$, and $C_{\varepsilon}^{\prime}$ are independent of $t$.

Consider the following integral:

$$
\begin{aligned}
& \int_{0}^{\infty} k(s)\left|\nabla \theta_{u}(t, s)\right|^{2} d s \\
= & \int_{0}^{\infty} k(s)|\nabla u(t)-\nabla u(t-s)|^{2} d s \\
= & \int_{0}^{\infty} k(s)|\nabla u(t)|^{2} d s-2 \int_{0}^{\infty} k(s)(\nabla u(t), \nabla u(t-s)) d s \\
& \quad+\int_{0}^{\infty} k(s)|\nabla u(t-s)|^{2} d s \\
\leq & \alpha|\nabla u(t)|^{2}+2 \int_{0}^{\infty} k(s)\left[\frac{|\nabla u(t)|^{2}}{4 \alpha}+|\nabla u(t-s)|^{2} \times \alpha\right] d s \\
& \quad+\int_{0}^{\infty} k(s)|\nabla u(t-s)|^{2} d s \\
= & \left(\alpha+\frac{1}{2}\right)|\nabla u(t)|^{2}+(1+2 \alpha) \int_{0}^{\infty} k(s)|\nabla u(t-s)|^{2} d s .
\end{aligned}
$$

(Recall that $\alpha=\int_{0}^{\infty} k(s) d s$.) Using this inequality in (3.41), we obtain

$$
\begin{aligned}
\int_{0}^{t} \tau\left|\partial_{t} u(\tau)\right|^{2} d \tau+\frac{t}{2}\left\{\left(\frac{1}{2}-\varepsilon\right)|\nabla u(t)|^{2}+\gamma_{1}\|u(t)\|_{L_{p}(\Omega)}^{p}\right\} \\
\leq \frac{t}{2}(1+2 \alpha) \int_{0}^{\infty} k(s)|\nabla u(t-s)|^{2} d s \\
+\gamma_{3} \int_{0}^{t}|\nabla u(\tau)|^{2} d \tau+\gamma_{2} \int_{0}^{t}\|u(\tau)\|_{L_{p}(\Omega)}^{p} d \tau \\
\quad+\frac{1}{2} \int_{0}^{t} \tau \int_{0}^{\infty} \mu(s)\left|\nabla \theta_{u}(\tau, s)\right|^{2} d s d \tau+C_{\varepsilon}^{\prime} t, \quad \forall t \geq 0 .
\end{aligned}
$$


Finally, we estimate the last integral in the right-hand side of (3.42) as follows:

$$
\begin{aligned}
& \int_{0}^{t} \tau \int_{0}^{\infty} \mu(s)\left|\nabla \theta_{u}(\tau, s)\right|^{2} d s d \tau \\
& \quad=\int_{0}^{t} \tau \int_{0}^{\infty} \mu(s)|\nabla u(\tau)-\nabla u(\tau-s)|^{2} d s d \tau \\
& \quad \leq 2 \int_{0}^{t} \tau \int_{0}^{\infty} \mu(s)\left(|\nabla u(\tau)|^{2}+|\nabla u(\tau-s)|^{2}\right) d s d \tau \\
& \quad=2 k(0) \int_{0}^{t} \tau|\nabla u(\tau)|^{2} d \tau+2 \int_{0}^{t} \tau \int_{0}^{\infty} \mu(s)|\nabla u(\tau-s)|^{2} d s d \tau \\
& \quad \leq c_{6}^{\prime} R^{2}+C_{6}^{\prime}\left(t+t^{2}\right) .
\end{aligned}
$$

In the last inequality, we have used estimates (3.26) and (3.27).

We now set $\varepsilon=\frac{1}{4}$ in (3.42), apply estimates (3.16), (3.18), and (3.43) to the right-hand side of (3.42), and deduce

$$
\int_{0}^{t} \tau\left|\partial_{t} u(\tau)\right|^{2} d \tau+\frac{t}{2}\left\{\frac{1}{4}|\nabla u(t)|^{2}+\gamma_{1}\|u(t)\|_{L_{p}(\Omega)}^{p}\right\} \leq c_{6}^{\prime \prime} R^{2}+C_{6}^{\prime \prime}\left(t+t^{2}\right) .
$$

Inequality (3.28) is completely proved.

\section{Trajectory and Global ATtractors For HEAT EQUATIONS WITH MEMORY}

We consider equations (2.1) and (2.2) with the initial datum (2.17). It is clear that this problem is of the form (1.1), with $E=L_{2}(\Omega)$. Concerning the phase space $\mathcal{E}^{-}$, we take

$$
\mathcal{E}^{-}=C_{b}\left(\mathbb{R}_{-} ; L_{2}(\Omega)\right) \cap L_{2}^{b}\left(\mathbb{R}_{-} ; H_{0}^{1}(\Omega)\right) .
$$

The space $\mathcal{E}^{-}$is a vector subspace of $C_{b}\left(\mathbb{R}_{-} ; L_{2}(\Omega)\right)$.

We assume that $v(\cdot) \in \mathcal{E}^{-}$. From Theorem 2.1, we conclude that, for every $v(\cdot) \in \mathcal{E}^{-}$, problem (2.1), (2.2), and (2.17) has a unique solution $u(t)$ such that

$$
u \in C_{b}\left(\mathbb{R}_{+} ; L_{2}(\Omega)\right) \cap L_{2}^{b}\left(\mathbb{R}_{+} ; H_{0}^{1}(\Omega)\right) \cap L_{p}^{\text {loc }}\left(\mathbb{R}_{+} ; L_{p}(\Omega)\right) .
$$

Thus, problem (2.1), (2.2), and (2.17) generates a semigroup in $\mathcal{E}^{-}$acting by the formula

$$
(S(t) v)(s)=u(t+s), \quad s \leq 0, t \geq 0,
$$

where $u(t)$ is the solution of (2.1)-(2.2) with initial datum $v(\cdot)$. It is obvious that the semigroup $\{S(t)\}$ maps the space $\mathcal{E}^{-}$into itself. 
We now construct the trajectory attractor for the semigroup $\{S(t)\}$. To measure the convergence to the attractor, we use the local uniform convergence topology of the space $C^{\text {loc }}\left(\mathbb{R}_{-} ; L_{2}(\Omega)\right)$, while the collection of bounded sets in $\mathcal{E}^{-}$is taken in the metric of this space itself:

$$
\mathcal{F}=\mathcal{E}^{-}=C_{b}\left(\mathbb{R}_{-} ; L_{2}(\Omega)\right) \cap L_{2}^{b}\left(\mathbb{R}_{-} ; H_{0}^{1}(\Omega)\right) .
$$

The norm in this space is defined as follows:

$$
\begin{aligned}
\|v\|_{\mathcal{F}}^{2} & =\|v\|_{C_{b}\left(\mathbb{R}_{-} ; L_{2}(\Omega)\right)}^{2}+\|v\|_{L_{2}^{b}\left(\mathbb{R}_{-} ; H_{0}^{1}(\Omega)\right)}^{2} \\
& =\sup _{t \leq 0}|v(t)|^{2}+\sup _{t \leq 0} \int_{t-1}^{t}|\nabla v(s)|^{2} d s .
\end{aligned}
$$

Proposition 4.1. The semigroup $\{S(t)\}$ associated with problem (2.1), (2.2), and (2.17) is uniformly bounded in $\mathcal{F}$ and, moreover, it has an attracting (in $C^{\text {loc }}\left(\mathbb{R}_{+} ; L_{2}(\Omega)\right)$ ) set which is bounded in $\mathcal{F}$.

Proof. We use Proposition 3.1. Let $B \subset \mathcal{E}^{-}$be a bounded set (for the norm (4.3)). Then, there exists a number $r>0$ such that

$$
\|v\|_{C_{b}\left(\mathbb{R}_{-} ; L_{2}(\Omega)\right)}^{2}+\|v\|_{L_{2}^{b}\left(\mathbb{R}_{-} ; H_{0}^{1}(\Omega)\right)}^{2} \leq r^{2}, \quad \forall v \in B .
$$

Therefore,

$$
|v(0)|^{2} \leq r^{2} \quad \text { and } \quad \int_{t-1}^{t}|\nabla v(s)|^{2} d s \leq r^{2}, \quad \forall t \leq 0 .
$$

Recall that $\varphi(t)=|u(t)|^{2}+\int_{0}^{\infty} \mu(s)\left|\nabla \eta_{u}(t, s)\right|^{2} d s$. We note that

$$
\begin{aligned}
\varphi(0) & =|v(0)|^{2}+\int_{0}^{\infty} \mu(s)\left|\nabla \eta_{v}(0, s)\right|^{2} d s \\
& =|v(0)|^{2}+\int_{0}^{\infty} \mu(s)\left|\left(\int_{-s}^{0} \nabla v(\tau) d \tau\right)\right|^{2} d s \\
& \leq r^{2}+\int_{0}^{\infty} \mu(s)\left(\int_{-s}^{0} 1 \times|\nabla v(\tau)| d \tau\right)^{2} d s \\
& \leq r^{2}+\int_{0}^{\infty} \mu(s) s\left(\int_{-s}^{0}|\nabla v(\tau)|^{2} d \tau\right) d s \\
& \leq r^{2}+\int_{0}^{\infty} \mu(s) s(s+1) r^{2} d s \\
& =\left(1+\int_{0}^{\infty} \mu(s) s(s+1) d s\right) r^{2}=c^{\prime} r^{2} .
\end{aligned}
$$


Here, we have used the estimate

$$
\begin{aligned}
\int_{-s}^{0}|\nabla v(\tau)|^{2} d \tau=\int_{-s}^{-[s]}+\cdots+\int_{-2}^{-1}+\int_{-1}^{0} & \leq([s]+1) r^{2} \\
& \leq(s+1) r^{2}
\end{aligned}
$$

Thus, inequality (4.4) implies that

$$
\varphi(0) \leq c^{\prime} r^{2} \equiv R^{2}, \quad \forall v \in B .
$$

Inequality (3.2) yields, on the segment $[t, t+1]$,

$$
\int_{t}^{t+1}|\nabla u(s)|^{2} d s \leq \varphi(t)+C_{0}, \quad \forall t \geq 0 .
$$

It follows from (3.1) that

$$
\varphi(t) \leq \varphi(0)+C_{0} / \varkappa \leq R^{2}+C_{0} / \varkappa .
$$

From (4.7) and (4.8), we conclude that

$$
\begin{gathered}
|u(t)|^{2} \leq \varphi(t) \leq R^{2}+C_{0} / \varkappa ; \\
\int_{t}^{t+1}|\nabla u(s)|^{2} d s \leq \varphi(t)+C_{0} \leq\left(R^{2}+C_{0} / \varkappa\right)+C_{0}, \quad \forall t \geq 0 .
\end{gathered}
$$

Therefore,

$$
\|S(t) v\|_{\mathcal{F}}^{2} \leq R_{1}^{2}=R_{1}^{2}(R), \quad \forall t \geq 0,
$$

where $R_{1}^{2}=2\left(R^{2}+C_{0} / \mathcal{\varkappa}\right)+C_{0}$, and the semigroup $\{S(t)\}$ is uniformly bounded in $\mathcal{F}$.

To prove the existence of a bounded attracting set, we use Corollary 3.2. Consider the following set in $\mathcal{E}^{-}$:

$$
P_{0}=\left\{\left.v \in \mathcal{E}^{-}\left|\sup _{s \leq 0}\right| v(s)\right|^{2} \leq 2 C_{0}^{\prime}, \sup _{s \leq 0} \int_{s-1}^{s}|\nabla v(\tau)|^{2} d \tau \leq 2 C_{0}^{\prime}\right\},
$$

where $C_{0}^{\prime}=C_{0} / \mathcal{\varkappa}+C_{0}($ see $(3.15))$. It is clear that $P_{0}$ is bounded in $\mathcal{F}$. We claim that $P_{0}$ is an attracting (in $C^{\text {loc }}\left(\mathbb{R}_{-} ; L_{2}(\Omega)\right)$ ) set. Indeed, we fix an arbitrary number $M>0$ and consider an arbitrary bounded set $B \subset \mathcal{E}^{-}$, that is, for some $r>0$,

$$
|v(s)|^{2} \leq r^{2} \quad \text { and } \quad \int_{s-1}^{s}|\nabla v(\tau)|^{2} d \tau \leq r^{2}, \quad \forall s \leq 0
$$


Then,

$$
\varphi(0) \leq R^{2}, \quad \forall v \in B\left(R^{2}=c^{\prime} r^{2}\right)
$$

and, concerning the corresponding solution $u(t)=u(t, v)$, we obtain

$$
\begin{aligned}
|u(t)|^{2} & \leq R^{2} e^{-\varkappa t}+C_{0}, \\
\int_{t-1}^{t}|\nabla u(\tau)|^{2} d \tau & \leq R^{2} e^{-\varkappa(t-1)}+C_{0}^{\prime}, \quad \forall t \geq 1
\end{aligned}
$$

(see (3.14) and (3.15)). We choose the number $t_{1}$ such that

$$
R^{2} e^{-\varkappa\left(t_{1}-M-1\right)} \leq C_{0}^{\prime}, \quad t_{1} \geq M .
$$

Then, for all $t \geq t_{1}, R^{2} e^{-\varkappa(t-M-1)}+C_{0}^{\prime} \leq 2 C_{0}^{\prime}$, and the set $S(t) B$, restricted to the segment $[-M, 0]$, belongs to the set $P_{0}$, also restricted to $[-M, 0]$. Therefore, $\operatorname{dist}_{C\left([-M, 0] ; L_{2}(\Omega)\right)}\left(S(t) B, P_{0}\right)=0$. Thus, $P_{0}$ attracts $S(t) B$ as $t \rightarrow+\infty$ in the topology of the space $C^{\text {loc }}\left(\mathbb{R}_{-} ; L_{2}(\Omega)\right)$.

Notice that the attracting set $P_{0}$ is not absorbing for the semigroup, i.e., we cannot state that $S(t) B \subseteq P_{0}$ when $t$ is large, since every solution $u(t)$ has a "tail," namely, $v(s), s \leq 0$. It is clear that

$$
\|v\|_{\mathcal{F}}^{2} \leq\|S(t) v\|_{\mathcal{F}}^{2}, \quad \forall t \geq 0 .
$$

So, if the weight of this "tail" is large, then the weight of $\|S(t) v\|_{\mathcal{F}}^{2}$ is large for all $t \geq 0$. This phenomenon reflects the peculiarity of equations with memory.

Proposition 4.2. The semigroup $\{S(t)\}$ has $\left(\right.$ in $\left.C^{\text {loc }}\left(\mathbb{R}_{+} ; L_{2}(\Omega)\right)\right)$ an attracting set which is compact in $C^{\text {loc }}\left(\mathbb{R}_{+} ; L_{2}(\Omega)\right)$ and bounded in $\mathcal{F}$.

Proof. Consider the following set in the space $\mathcal{E}^{-}$:

$$
P_{1}=\left\{\left.v \in \mathcal{E}^{-}\left|\varphi(0) \leq R_{1}^{2}, \int_{0}^{\infty} e^{-\varkappa s}\right| \nabla v(-s)\right|^{2} d s \leq R_{1}^{2}\right\},
$$

where $R_{1}^{2}=2 \max \left\{C_{0} / \varkappa, C_{3}\right\}$ (see (3.14) and (3.20)). The set $P_{1}$ is absorbing for the semigroup $\{S(t)\}$. Indeed, let $B$ be a bounded set in $\mathcal{E}^{-}$, i.e., let $B$ satisfy (4.4) and (4.5) for some number $r$. Then, estimate (4.6) holds. Furthermore, we have

$$
\begin{aligned}
\int_{0}^{\infty} e^{-\varkappa s}|\nabla v(-s)|^{2} d s \\
\quad=\int_{0}^{1} e^{-\varkappa s}|\nabla v(-s)|^{2} d s+\int_{1}^{2} e^{-\varkappa s}|\nabla v(-s)|^{2} d s+\cdots \\
\quad \leq r^{2}+r^{2} e^{-\varkappa}+r^{2} e^{-2 \varkappa}+\cdots=\frac{r^{2}}{1-e^{-\varkappa}}=c^{\prime \prime} r^{2} .
\end{aligned}
$$


We set $R^{2}=\left(c^{\prime}+c^{\prime \prime}\right) r^{2}$. Hence, for any $v \in B$,

$$
\varphi(0) \leq R^{2}, \quad \int_{0}^{\infty} e^{-\varkappa s}|\nabla v(-s)|^{2} d s \leq R^{2} .
$$

We now choose the number $t_{1}=t_{1}(r)$ so that

$$
R^{2} e^{-\varkappa t_{1}} \leq \frac{C_{0}}{\varkappa}, \quad c_{3} R^{2}\left(1+t_{1}\right) e^{-\varkappa t_{1}} \leq C_{3} .
$$

From (3.14) and (3.20), we then conclude that, for all $t \geq t_{1}$,

$$
\varphi(t) \leq R_{1}^{2}, \quad \int_{0}^{\infty} e^{-\varkappa s}|\nabla u(t-s)|^{2} d s \leq R_{1}^{2},
$$

where $R_{1}^{2}=2 \max \left\{C_{0} / \varkappa, C_{3}\right\}$ and $u(t)$ is the corresponding solution of (2.1)(2.2). Therefore, $S(t) B \subseteq P_{1}$ for $t \geq t_{1}$, i.e., $P_{1}$ is an absorbing set of the semigroup $\{S(t)\}$.

We now construct a compact attracting set. We shall use Proposition 3.5. For every $v \in P_{1}$, inequality (3.17) holds with $R=R_{1}$. It follows from (3.28) that the corresponding solution $u(\cdot)$ satisfies the inequality $(t=2)$

$$
\int_{0}^{2} \tau\left|\partial_{t} u(\tau)\right|^{2} d \tau+2|\nabla u(2)|^{2}+2|| u(2) \|_{L_{p}(\Omega)}^{p} \leq c_{6} R_{1}^{2}+4 C_{6}=R_{2}^{2}
$$

In particular,

$$
\int_{1}^{2}\left|\partial_{t} u(\tau)\right|^{2} d \tau \leq R_{2}^{2}, \quad|\nabla u(2)|^{2} \leq R_{2}^{2}, \quad\|u(2)\|_{L_{p}(\Omega)}^{p} \leq R_{2}^{2} .
$$

Thus, for any element $w \in S(2) P_{1}$, we have the inequalities

$$
\int_{-1}^{0}\left|\partial_{t} w(\tau)\right|^{2} d \tau \leq R_{2}^{2}, \quad|\nabla w(0)|^{2} \leq R_{2}^{2}, \quad\|w(0)\|_{L_{p}(\Omega)}^{p} \leq R_{2}^{2} .
$$

Consider the set

$$
\begin{aligned}
& P_{2}=\left\{\left.v \in \mathcal{E}^{-}\left|\int_{h-1}^{h}\right| \partial_{t} v(\tau)\right|^{2} d \tau\right. \leq R_{2}^{2}, \\
&\left.|\nabla v(h)|^{2} \leq R_{2}^{2},\|v(h)\|_{L_{p}(\Omega)}^{p} \leq R_{2}^{2}, \forall h \leq 0\right\} .
\end{aligned}
$$

It is clear that $P_{2}$ is bounded in $\mathcal{F}$. It follows from Lemmas 1.7 and 1.8 that the set $P_{2}$ is compact in $C^{\text {loc }}\left(\mathbb{R}_{-} ; L_{2}(\Omega)\right.$ ) (we apply Lemma 1.8 with $E=L_{2}(\Omega)$, $E_{1}=H_{0}^{1}(\Omega), E^{\prime}=L_{2}(\Omega)$, and $\left.p=2\right)$. 
We claim that the set $P_{2}$ is attracting for $\{S(t)\}$ in the topology of the space $C^{\text {loc }}\left(\mathbb{R}_{-} ; L_{2}(\Omega)\right)$. Consider an arbitrary bounded set $B$, whose norm does not exceed some number $r$ (see (4.4) and (4.5)). We fix a number $M>0$. Consider the solution $u(\cdot)$ corresponding to an arbitrary function $v \in B$. We note that, for $t \geq t_{2}=t_{1}+M$,

$$
\begin{aligned}
& \varphi(t+h) \leq R_{1}^{2}, \\
& \int_{0}^{\infty} e^{-\varkappa s}|\nabla u(t+h-s)|^{2} d s \leq R_{1}^{2}, \\
& S(t+h) B \subseteq P_{1}, \quad \forall h \in[-M, 0],
\end{aligned}
$$

where $t_{1}$ is defined in (4.10). Therefore, the function $u(\cdot+t+h)$ satisfies (4.13) for all $h \in[-M, 0]$ and, for all $t \geq t_{2}$ :

$$
\begin{aligned}
\int_{1}^{2}\left|\partial_{t} u(\tau+t+h)\right|^{2} d \tau & \leq R_{2}^{2}, \\
|\nabla u(t+h+2)|^{2} & \leq R_{2}^{2}, \\
\|u(t+h+2)\|_{L_{p}(\Omega)}^{p} & \leq R_{2}^{2} .
\end{aligned}
$$

Hence, the set $S(2+t) B=S(2) S(t) B$ satisfies the following property: for any $w \in S(2+t) B$,

$$
\begin{aligned}
\int_{h-1}^{h}\left|\partial_{t} w(\tau)\right|^{2} d \tau & \leq R_{2}^{2}, \\
|\nabla w(h)|^{2} & \leq R_{2}^{2}, \\
\|w(h)\|_{L_{p}(\Omega)}^{p} & \leq R_{2}^{2}, \quad \forall h \in[-M, 0] .
\end{aligned}
$$

Thus, for all $t \geq t_{2}$, the set $S(2+t) B$, restricted to the segment $[-M, 0]$, belongs to the set $P_{2}$, also restricted to the segment $[-M, 0]$. Therefore,

$$
\operatorname{dist}_{C\left([-M, 0] ; L_{2}(\Omega)\right)}\left(S(t) B, P_{2}\right)=0, \quad \forall t \geq t_{2} .
$$

Thus, the set $P_{2}$ attracts $S(t) B$ as $t \rightarrow+\infty$ in the topology of $C^{\text {loc }}\left(\mathbb{R}_{-} ; L_{2}(\Omega)\right)$. This finishes the proof of the proposition.

Summarizing, we have defined the semigroup $\{S(t)\}$ acting in the space $\mathcal{E}^{-}$and generated by the equation with memory (2.1). We have constructed a bounded (in $\mathcal{F}=\mathcal{E}^{-}$) and compact (in $C^{\text {loc }}\left(\mathbb{R}_{-} ; L_{2}(\Omega)\right)$ ) attracting set. Thus, Theorems 1.5 and 1.11 are applicable and we have the following result.

Theorem 4.3. The semigroup $\{S(t)\}$ associated with (2.1)-(2.2) possesses the trajectory $\left(\mathcal{E}^{-}, C^{\text {loc }}\left(\mathbb{R}_{-} ; L_{2}(\Omega)\right)\right)$-attractor $\mathfrak{A}$ and the global $\left(\mathcal{E}^{-}, L_{2}(\Omega)\right)$-attractor $\mathcal{A}$ such that

$$
\mathcal{A}=\mathfrak{A}(0) .
$$


We note that $\mathfrak{A} \subseteq P_{2}$ (see (4.15)). Corollary 1.6 and this observation imply the following result.

Corollary 4.4. The trajectory attractor $\mathfrak{A}$ consists of the functions $v(t), t \leq 0$, which are bounded in $\mathcal{E}^{-}$and satisfy equation (2.1) for all $t \leq 0$. Moreover, the set $\mathfrak{A}$ is bounded in the space $L_{\infty}\left(\mathbb{R}_{-} ; H_{0}^{1}(\Omega) \cap L_{p}(\Omega)\right)$ and the set $\left\{\partial_{t} v \mid v \in\right.$ $\mathfrak{A}\}$ is bounded in $L_{2}^{b}\left(\mathbb{R}_{-} ; L_{2}(\Omega)\right)$. Finally, the global attractor $\mathcal{A}$ is bounded in $H_{0}^{1}(\Omega) \cap L_{p}(\Omega)$.

Corollary 4.5. The trajectory attractor $\mathfrak{A}$ is compact in $C^{\mathrm{loc}}\left(\mathbb{R}_{-} ; H^{1-\varepsilon}(\Omega)\right)$ for every $\varepsilon \in(0,1]$ and, moreover, it attracts the bounded sets of $\mathcal{E}^{-}$in the topology of $C^{\mathrm{loc}}\left(\mathbb{R}_{-} ; H^{1-\varepsilon}(\Omega)\right)$ in the following sense: for any bounded set $B \subset \mathcal{E}^{-}$and for any $M>0$,

$$
\operatorname{dist}_{C\left([-M, 0] ; H^{1-\varepsilon}(\Omega)\right)}(S(t) B, \mathfrak{A}) \rightarrow 0 \quad(t \rightarrow+\infty) .
$$

In particular, for $M=0$,

$$
\sup _{v \in B} \operatorname{dist}_{H^{1-\varepsilon}(\Omega)}(u(t), \mathcal{A}) \rightarrow 0 \quad(t \rightarrow+\infty),
$$

where $u(t)$ is the solution of $(2.1)-(2.2)$ with initial datum $v(\cdot) \in B$.

The proof of this assertion is based on the fact that the set $P_{2}$ is compact in the space $C^{\text {loc }}\left(\mathbb{R}_{-} ; H^{1-\varepsilon}(\Omega)\right.$ ) (which follows from Lemmas 1.7 and 1.8). We leave the details to the reader.

\section{SMOOTHNESS AND CONNECTEDNESS OF THE TRAJECTORY ATTRACTOR}

In this section, we shall prove that the trajectory attractor satisfies some additional smoothness properties and that it is a connected set.

We shall need one more result concerning the smoothness of the solutions of equation (2.1).

Proposition 5.1. Let the initial datum $v(\cdot)$ in (2.17) satisfy (3.17). Then, the corresponding solution $u(\cdot)$ of (2.1)-(2.2) satisfies the inequality

$$
t^{2}\left|\partial_{t} u(t)\right|^{2}+\int_{0}^{t} \tau^{2}\left|\nabla \partial_{t} u(\tau)\right|^{2} d \tau \leq c_{7}(1+t) R^{2}+C_{7}\left(t+t^{3}\right), \quad \forall t>0 .
$$

Proof. As usual, we prove (5.1) for the Faedo-Galerkin approximation systems (2.18) and obtain the same estimate for the exact solution by passing to the limit as $N \rightarrow \infty$. We shall omit the index $N$.

We rewrite equation (2.18) as follows (see (2.20)):

$$
\begin{aligned}
& \partial_{t} u(t)=\Delta u(t)+\int_{0}^{\infty} \mu(s) \Delta \eta_{u}(t, s) d s-P f(u(t))+P g, \\
& \left.u\right|_{\partial \Omega}=0,
\end{aligned}
$$


where

$$
\eta_{u}(t, s)=\int_{t-s}^{t} u(\tau) d \tau
$$

It follows from (5.3) that

$$
\partial_{t} \eta_{u}(t, s)=u(t)-u(t-s) \equiv \theta_{u}(t, s) \quad(\text { see (3.29)) } .
$$

We now differentiate equation (5.2) in $t$ and set $\bar{u}(t)=\partial_{t} u(t)$. We have the following equation for the function $\bar{u}(t)$, taking into account (5.4):

$$
\begin{aligned}
& \partial_{t} \bar{u}(t)=\Delta \bar{u}(t)+\int_{0}^{\infty} \mu(s) \Delta \theta_{u}(t, s) d s-P f^{\prime}(u(t)) \bar{u}(t), \\
& \left.\bar{u}\right|_{\partial \Omega}=0 .
\end{aligned}
$$

We multiply Equation (5.5) with $\bar{u}(t)$ and integrate over $\Omega$. Performing classical transformations, we obtain

$$
\begin{aligned}
\frac{1}{2} \frac{d}{d t}|\bar{u}(t)|^{2} & +|\nabla \bar{u}(t)|^{2} \\
& =\int_{0}^{\infty} \mu(s)\left(\Delta \theta_{u}(t, s), \bar{u}(t)\right) d s-\left(f^{\prime}(u(t)) \bar{u}(t), \bar{u}(t)\right)
\end{aligned}
$$

Recall that the function $\bar{u}(t)$ satisfies the identity

$$
\bar{u}(t)=\partial_{t} u(t)=\partial_{t} \theta(t, s)+\partial_{s} \theta(t, s)
$$

(see also (3.31)). Therefore, the integral in the right-hand side of (5.6) is equal to

$$
\begin{aligned}
& \int_{0}^{\infty} \mu(s)\left(\Delta \theta_{u}(t, s), \partial_{t} \theta(t, s)+\partial_{s} \theta(t, s)\right) d s \\
&=-\frac{1}{2} \frac{d}{d t} \int_{0}^{\infty} \mu(s)\left|\nabla \theta_{u}(t, s)\right|^{2} d s+\frac{1}{2} \int_{0}^{\infty} \mu^{\prime}(s)\left|\nabla \theta_{u}(t, s)\right|^{2} d s .
\end{aligned}
$$

Here, we have used transformations (3.33)-(3.34), where, obviously, due to (2.9), we can replace the kernel $k(s)$ by the kernel $\mu(s)$. Hence, from (5.6), we conclude that

$$
\begin{aligned}
& \frac{1}{2} \frac{d}{d t}\left\{|\bar{u}(t)|^{2}+\int_{0}^{\infty} \mu(s)\left|\nabla \theta_{u}(t, s)\right|^{2} d s\right\} \\
& \quad+|\nabla \bar{u}(t)|^{2}-\frac{1}{2} \int_{0}^{\infty} \mu^{\prime}(s)\left|\nabla \theta_{u}(t, s)\right|^{2} d s=-\left(f^{\prime}(u(t)) \bar{u}(t), \bar{u}(t)\right) .
\end{aligned}
$$


Inequality (2.3) implies

$$
-\left(f^{\prime}(u(t)) \bar{u}(t), \bar{u}(t)\right) \leq D|\bar{u}(t)|^{2} .
$$

Furthermore, it follows from (2.8) and (2.7) that $-\mu^{\prime}(s) \geq 0$. Thus, from (5.9), we obtain the inequality

$$
\frac{1}{2} \frac{d}{d t}\left\{|\bar{u}(t)|^{2}+\int_{0}^{\infty} \mu(s)\left|\nabla \theta_{u}(t, s)\right|^{2} d s\right\}+|\nabla \bar{u}(t)|^{2} \leq D|\bar{u}(t)|^{2} .
$$

We multiply this inequality with $t^{2}$ :

$$
\begin{aligned}
\frac{1}{2} \frac{d}{d t}\left\{t^{2}|\bar{u}(t)|^{2}\right. & \left.+t^{2} \int_{0}^{\infty} \mu(s)\left|\nabla \theta_{u}(t, s)\right|^{2} d s\right\}+t^{2}|\nabla \bar{u}(t)|^{2} \\
& \leq t|\bar{u}(t)|^{2}+t \int_{0}^{\infty} \mu(s)\left|\nabla \theta_{u}(t, s)\right|^{2} d s+t^{2} D|\bar{u}(t)|^{2} .
\end{aligned}
$$

Integrating $(5.11)$ over $[0, t]$, we have

$$
\begin{aligned}
& \frac{1}{2} t^{2}|\bar{u}(t)|^{2}+\frac{1}{2} t^{2} \int_{0}^{\infty} \mu(s)\left|\nabla \theta_{u}(t, s)\right|^{2} d s+\int_{0}^{t} \tau^{2}|\nabla \bar{u}(\tau)|^{2} d \tau \\
& \leq \int_{0}^{t} \tau|\bar{u}(\tau)|^{2} d \tau+\int_{0}^{t} \tau \int_{0}^{\infty} \mu(s)\left|\nabla \theta_{u}(\tau, s)\right|^{2} d s d \tau+D \int_{0}^{t} \tau^{2}|\bar{u}(t)|^{2} d \tau .
\end{aligned}
$$

Notice that $\mu(s) \geq 0$ for all $s \geq 0$. Thus, (5.12) implies

$$
\begin{aligned}
& t^{2}|\bar{u}(t)|^{2}+2 \int_{0}^{t} \tau^{2}|\nabla \bar{u}(\tau)|^{2} d \tau \\
& \quad \leq 2(1+D t) \int_{0}^{t} \tau\left|\partial_{t} u(\tau)\right|^{2} d \tau+2 \int_{0}^{t} \tau \int_{0}^{\infty} \mu(s)\left|\nabla \theta_{u}(\tau, s)\right|^{2} d s d \tau
\end{aligned}
$$

We estimate the first integral in the right-hand side of (5.13) by using (3.28). We also apply inequalities (3.27) and (3.43) and deduce

$$
\begin{aligned}
& t^{2}|\bar{u}(t)|^{2}+2 \int_{0}^{t} \tau^{2}|\nabla \bar{u}(\tau)|^{2} d \tau \leq 2(1+D t)\left(c_{6} R^{2}+C_{6} t^{2}\right) \\
&+2 c_{6}^{\prime} R^{2}+2 C_{6}^{\prime}\left(t+t^{2}\right) \leq c_{7}^{\prime}(1+t) R^{2}+C_{7}^{\prime}\left(t+t^{3}\right) .
\end{aligned}
$$

Consequently,

$$
t^{2}\left|\partial_{t} u(t)\right|^{2}+\int_{0}^{t} \tau^{2}\left|\nabla \partial_{t} u(\tau)\right|^{2} d \tau \leq c_{7}(1+t) R^{2}+C_{7}\left(t+t^{3}\right) .
$$

This finishes the proof of the proposition. 
Corollary 5.2. The trajectory attractor $\mathfrak{A}$ associated with problem (2.1)-(2.2) is bounded in $C_{b}\left(\mathbb{R}_{-} ; H_{0}^{1}(\Omega)\right) \cap L_{\infty}\left(\mathbb{R}_{-} ; L_{p}(\Omega)\right)$ and the set $\left\{\partial_{t} v \mid v \in \mathfrak{A}\right\}$ is bounded in $L_{\infty}\left(\mathbb{R}_{-} ; L_{2}(\Omega)\right) \cap L_{2}^{b}\left(\mathbb{R}_{-} ; H_{0}^{1}(\Omega)\right)$.

We now prove that the trajectory attractor $\mathfrak{A}$ is connected in the space $L_{2}^{\text {loc }}\left(\mathbb{R}_{-} ; L_{2}(\Omega)\right)$. We recall that, for equations without memory, the connectedness of the global attractors is usually proved by using the continuity of the corresponding semigroups in the phase spaces of initial data (see, for instance, [19]). However, in our example, the semigroup $\{S(t)\}$ acting in the phase space $\mathcal{E}^{-}=C_{b}\left(\mathbb{R}_{-} ; L_{2}(\Omega)\right) \cap L_{2}^{b}\left(\mathbb{R}_{-} ; H_{0}^{1}(\Omega)\right)$ is not continuous in the topology of $L_{2}^{\text {loc }}\left(\mathbb{R}_{-} ; L_{2}(\Omega)\right)$. This fact is easy to show for the linear homogeneous equation with memory (2.1), where $f \equiv 0, g \equiv 0$, by considering an initial datum $v$ with small norm in $L_{2}^{\text {loc }}\left(\mathbb{R}_{-} ; L_{2}(\Omega)\right)$ and with large norm in $L_{2}^{\text {loc }}\left(\mathbb{R}_{-} ; H_{0}^{1}(\Omega)\right)$. Then, the norm of $S(t) v$ in $L_{2}^{\text {loc }}\left(\mathbb{R}_{-} ; L_{2}(\Omega)\right)$ is also large. Nevertheless, the semigroup $\{S(t)\}$ is continuous from $L_{2}^{\text {loc }}\left(\mathbb{R}_{-} ; H_{0}^{1}(\Omega)\right)$ into $L_{2}^{\text {loc }}\left(\mathbb{R}_{-} ; L_{2}(\Omega)\right)$. This observation allows to prove the connectedness of the trajectory attractor.

Proposition 5.3. Let $B \subset \mathcal{E}^{-}$be a bounded set in $C_{b}\left(\mathbb{R}_{-} ; H_{0}^{1}(\Omega)\right)$, i.e.,

$$
|\nabla v(s)|^{2} \leq R^{2}, \quad \forall s \leq 0, \forall v \in B
$$

Then, the mapping $S(t): B \rightarrow \mathcal{E}^{-}$is continuous from $L_{2}^{\text {loc }}\left(\mathbb{R}_{-} ; H_{0}^{1}(\Omega)\right)$ into $L_{2}^{\text {loc }}\left(\mathbb{R}_{-} ; L_{2}(\Omega)\right)$.

Proof. We shall use estimate (2.36) from Theorem 2.1, where we proved the uniqueness of the solution of problem (2.1), (2.2), and (2.17). Let $u_{1}(t)$ and $u_{2}(t)$ be two solutions of problem (2.1), (2.2), and (2.17) with initial data $v_{1}(\cdot)$ and $v_{2}(\cdot)$, respectively. Repeating the reasoning leading from (2.31) to (2.36), we obtain that the difference $u_{1}(t)-u_{2}(t) \equiv w(t)$ satisfies the inequality

$$
|w(t)|^{2} \leq|w(0)|^{2}+\int_{0}^{\infty} \mu(s)\left|\nabla \eta_{w}(0, s)\right|^{2} d s+2 D \int_{0}^{t}|w(\tau)|^{2} d \tau
$$

$\forall t \geq 0$,

where $\eta_{w}(0, s)=\int_{-s}^{0} w(\tau) d \tau=\int_{-s}^{0}\left(v_{1}(\tau)-v_{2}(\tau)\right) d \tau, s \geq 0$. We note that

$$
\left|\nabla \eta_{w}(0, s)\right|^{2} \leq s\left(\sup _{\tau \in[-s, 0]}|\nabla w(\tau)|^{2}\right), \quad \forall s \geq 0
$$


Therefore, for a fixed $L>0$, we have

$$
\int_{0}^{\infty} \mu(s)\left|\nabla \eta_{w}(0, s)\right|^{2} d s=\int_{0}^{L}+\int_{L}^{\infty}
$$

$\leq\left(\int_{0}^{L} \mu(s) s d s\right)\left(\sup _{\tau \in[-L, 0]}|\nabla w(\tau)|^{2}\right)+\mu(0)\left(\int_{L}^{\infty} s e^{-\delta s} d s\right)\left(\sup _{\tau \leq-L}|\nabla w(\tau)|^{2}\right)$

$\leq\left(\int_{0}^{\infty} \mu(s) s d s\right)\left(\sup _{\tau \in[-L, 0]}|\nabla w(\tau)|^{2}\right)+\mu(0) \delta^{-2}\left(L \delta e^{-L \delta}+e^{-L \delta}\right)\left(\sup _{\tau \leq-L}|\nabla w(\tau)|^{2}\right)$

$\leq C^{\prime}\left\|v_{1}-v_{2}\right\|_{C\left([-L, 0] ; H_{0}^{1}(\Omega)\right)}^{2}+C^{\prime \prime} R^{2}(L \delta+1) e^{-\delta L}$,

where we assume that $v_{1}, v_{2} \in B$ and $B$ satisfies inequality (5.14).

We now fix arbitrary $M>0$ and $\varepsilon_{1}>0$. We choose the number $L=L\left(M, \varepsilon_{1}\right)$ such that

$$
C^{\prime \prime} R^{2}(L \delta+1) e^{-\delta L} \leq \frac{\varepsilon_{1}}{2}, \quad L \geq M .
$$

Let $v_{1}, v_{2}$ satisfy the inequality

$$
\left\|v_{1}-v_{2}\right\|_{C\left([-L, 0] ; H_{0}^{1}(\Omega)\right)}^{2} \leq \frac{\varepsilon_{1}}{2 C^{\prime}} .
$$

Then, due to (5.17),

$$
\int_{0}^{\infty} \mu(s)\left|\nabla \eta_{w}(0, s)\right|^{2} d s \leq \varepsilon_{1}
$$

Inserting this estimate into (5.15) and applying the Gronwall lemma to the resulting inequality, we obtain

$$
\begin{aligned}
\left|u_{1}(t)-u_{2}(t)\right|^{2}=|w(t)|^{2} & \leq\left(\left|v_{1}(0)-v_{2}(0)\right|^{2}+\varepsilon_{1}\right) e^{2 D t} \\
& \leq C \varepsilon_{1} e^{2 D t}, \quad \forall t \geq 0 .
\end{aligned}
$$

Thus, for any fixed $M>0$,

$$
\left\|S(t) v_{1}-S(t) v_{2}\right\|_{C\left([-M, 0] ; L_{2}(\Omega)\right)}^{2} \leq C \varepsilon_{1} e^{2 D t} .
$$

This means that, for any $M>0$, we can make the left-hand side of (5.19) arbitrary small, taking $\varepsilon_{1}$ sufficiently small and $L$ sufficiently large. Consequently, the mapping $S(t)$ is continuous from $L_{2}^{\text {loc }}\left(\mathbb{R}_{-} ; H_{0}^{1}(\Omega)\right)$ into $L_{2}^{\text {loc }}\left(\mathbb{R}_{-} ; L_{2}(\Omega)\right)$ on any bounded set $B$ in $C_{b}\left(\mathbb{R}_{-} ; H_{0}^{1}(\Omega)\right)$.

Theorem 5.4. The trajectory attractor $\mathfrak{A}$ is connected in the space $L_{2}^{\text {loc }}\left(\mathbb{R}_{-} ; L_{2}(\Omega)\right)$. 
Proof. We consider the attracting set $P_{2}$ constructed in Proposition 4.2 (see (4.15)). It follows easily from Proposition 5.1 that the set $P_{2}$ is bounded in the space $C_{b}\left(\mathbb{R}_{-} ; H_{0}^{1}(\Omega)\right)$. Then, Proposition 5.3 implies that each mapping $S(t)$, $t \geq 0$, is continuous on $P_{2}$ from $L_{2}^{\text {loc }}\left(\mathbb{R}_{-} ; H_{0}^{1}(\Omega)\right)$ into $L_{2}^{\text {loc }}\left(\mathbb{R}_{-} ; L_{2}(\Omega)\right)$. The set $P_{2}$ is clearly connected in $L_{2}^{\text {loc }}\left(\mathbb{R}_{-} ; H_{0}^{1}(\Omega)\right)$ and a continuous image of a connected set is a connected set. Therefore, the set $S(h) P_{2}$ is connected in $L_{2}^{\text {loc }}\left(\mathbb{R}_{-} ; L_{2}(\Omega)\right)$ for any $h \geq 0$. Notice that

$$
\mathfrak{A}=\bigcap_{h \geq 0} S(h) P_{2} \quad(\text { see }[12]) .
$$

It is known that the intersection of any collection of connected sets is a connected set as well. Thus, $\mathfrak{A}$ is connected in the space $L_{2}^{\text {loc }}\left(\mathbb{R}_{-} ; L_{2}(\Omega)\right)$ and the theorem is proved.

Corollary 5.5. The global attractor $\mathcal{A}$ is connected in $L_{2}(\Omega)$.

\section{Lyapunov Functions for the Heat Equation With Memory}

It is well-known that the heat equation (2.1) without memory, i.e., $k(s)=0$, for all $s \geq 0$, has a regular structure (see $[1,12,13])$. This property is closely connected with the existence of a global Lyapunov function for this equation. Recall that a global Lyapunov function is a functional defined on the phase space of the equation which strictly decays along any nonstationary solution of this equation. For the heat equation without memory, a choice of a Lyapunov function reads

$$
\Phi_{0}(v(\cdot))=\frac{1}{2}|\nabla v|^{2}+\int_{\Omega} F(v(x)) d x-\int_{\Omega} g(x) v(x) d x,
$$

where $v \in H_{0}^{1}(\Omega) \cap L_{p}(\Omega), F(v)=\int_{0}^{v} f(z) d z$. It is easy to show (see, for instance, [12]) that any solution $u(t)$ of this equation satisfies the equality

$$
\Phi_{0}\left(u\left(t_{2}\right)\right)-\Phi_{0}\left(u\left(t_{1}\right)\right)=-\int_{t_{1}}^{t_{2}}\left|\partial_{t} u(\tau)\right|^{2} d \tau, \quad \forall t_{2}>t_{1}>0 .
$$

This identity implies that the functional $\Phi_{0}(\cdot)$ is a global Lyapunov function for the heat equation without memory.

To prove (6.2), one multiplies the equation by $\partial_{t} u(t)$, integrates over $\Omega$, integrates by parts, and makes standard transformations. If we now perform a similar procedure for the equation with memory (2.1), we obtain the following. 
Let $u(t)$ be a solution of problem (2.1), (2.2), and (2.17), where $v(\cdot) \in \mathcal{E}^{-}$. Consider the following functional on $\mathcal{F}^{-}$:

$$
\begin{aligned}
\Phi_{k}(v(\cdot))=\frac{1}{2}(1+\alpha) \mid \nabla v & \left.(0)\right|^{2}+\int_{\Omega} F(v(x, 0)) d x \\
& -\int_{\Omega} g(x) v(x, 0) d x-\int_{0}^{\infty} k(s)\left|\nabla \theta_{v}(0, s)\right|^{2} d s,
\end{aligned}
$$

where $\alpha=\int_{0}^{\infty} k(s) d s, \theta_{v}(0, s)=v(\cdot, 0)-v(\cdot,-s)$. Repeating formally the transformations (3.30)-(3.35), we obtain the equality

$$
\left|\partial_{t} u(t)\right|^{2}+\frac{d}{d t} \Phi_{k}(S(t) v)=\frac{1}{2} \int_{0}^{\infty} \mu(s)\left|\nabla \theta_{u}(t, s)\right|^{2} d s,
$$

where $(S(t) v)(s)=u(t+s), t \geq 0, s \leq 0$, is the semigroup corresponding to problem (2.1), (2.2), and (2.17) and acting in $\mathcal{E}^{-}$. Integrating (6.3) in time, we have the identity

$$
\begin{aligned}
\Phi_{k}\left(S\left(t_{2}\right) v\right)-\Phi_{k}( & \left.S\left(t_{1}\right) v\right)=-\int_{t_{1}}^{t_{2}}\left|\partial_{t} u(\tau)\right|^{2} d \tau \\
& +\frac{1}{2} \int_{t_{1}}^{t_{2}} \int_{0}^{\infty} \mu(s)\left|\nabla \theta_{u}(\tau, s)\right|^{2} d s d \tau, \quad \forall t_{2}>t_{1}>0,
\end{aligned}
$$

which is similar to (6.2). Unfortunately, in (6.4), $\mu(s) \geq 0$ for all $s \geq 0$. Therefore, the functional $\Phi_{k}(S(t) v)$ may not decay in $t$. Thus, this does not lead to a Lyapunov function for the equation with memory.

Nevertheless, this obstacle can be partially removed by adding an auxiliary term coming from identity $(5.9)$ to $\Phi_{k}(\cdot)$. Then, we can construct a global Lyapunov function under the condition that the number $D$ from estimate (2.3) is less than the sum $\lambda_{1}+\delta$, where $\lambda_{1}$ is the first eigenvalue of the laplacian and the number $\delta$ is taken from (2.7). We now formulate this assertion precisely.

Recall that the memory kernel $\mu(s)$ in (6.4) satisfies inequality (2.7), i.e.,

$$
\mu^{\prime}(s)+\delta \mu(s) \leq 0, \quad \forall s \geq 0 .
$$

Consider the following functional:

$$
J(v(\cdot))=\delta \Phi_{k}(v)+\left|\partial_{t} v(0)\right|^{2}+\int_{0}^{\infty} \mu(s)\left|\nabla \theta_{v}(0, s)\right|^{2} d s .
$$

Theorem 6.1. Let the nonlinear function $f(u)$ satisfy the inequality

$$
f^{\prime}(u) \geq-D>-\lambda_{1}-\delta, \quad \forall u \in \mathbb{R} .
$$


Then, any solution $u(\cdot)$ of problem (2.1), (2.2), and (2.17) satisfies the inequality

$$
J\left(S\left(t_{2}\right) v\right)-J\left(S\left(t_{1}\right) v\right) \leq-\rho \int_{t_{1}}^{t_{2}}\left|\partial_{t} u(\tau)\right|^{2} d \tau, \quad \forall t_{2}>t_{1}>0,
$$

where $\rho=\lambda_{1}+\delta-D>0$.

Proof. From Propositions 3.5 and 5.1, it follows that the function $J(S(t) v)$ has a sense for all $t>0$ and $\partial_{t} u(\cdot) \in C_{b}\left([\varepsilon,+\infty) ; L_{2}(\Omega)\right) \cap L_{2}^{b}\left([\varepsilon,+\infty) ; H_{0}^{1}(\Omega)\right)$, for every $\varepsilon>0$. As usual, we first prove the analogue of (6.8) for the FaedoGalerkin approximations. Then, passing to the limit, we shall obtain (6.8) for the exact solution.

We proved in Proposition 3.5 that any Faedo-Galerkin approximation $u_{N}(t)=u(t)$ satisfies (3.35), that is,

$$
\frac{d}{d t} \Phi_{k}(S(t) v)=-\left|\partial_{t} u(t)\right|^{2}+\frac{1}{2} \int_{0}^{\infty} \mu(s)\left|\nabla \theta_{u}(t, s)\right|^{2} d s, \quad t>0 .
$$

At the same time, in the proof of Proposition 5.1, equality (5.9) was established, i.e.,

$$
\begin{aligned}
& \text { (6.10) } \frac{1}{2} \frac{d}{d t}\left\{\left|\partial_{t} u(t)\right|^{2}+\int_{0}^{\infty} \mu(s)\left|\nabla \theta_{u}(t, s)\right|^{2} d s\right\} \\
& =-\left|\nabla \partial_{t} u(t)\right|^{2}+\frac{1}{2} \int_{0}^{\infty} \mu^{\prime}(s)\left|\nabla \theta_{u}(t, s)\right|^{2} d s-\left(f^{\prime}(u(t)) \partial_{t} u(t), \partial_{t} u(t)\right),
\end{aligned}
$$

We now multiply equation (6.9) by $\delta$ and add the resulting equation to (6.10). We have the identity

$$
\begin{aligned}
\frac{d}{d t}\left\{\delta \Phi_{k}(S(t) v)+\right. & \left.\frac{1}{2}\left|\partial_{t} u(t)\right|^{2}+\frac{1}{2} \int_{0}^{\infty} \mu(s)\left|\nabla \theta_{u}(t, s)\right|^{2} d s\right\} \\
=-\left|\nabla \partial_{t} u(t)\right|^{2} & +\frac{1}{2} \int_{0}^{\infty}\left(\mu^{\prime}(s)+\delta \mu(s)\right)\left|\nabla \theta_{u}(t, s)\right|^{2} d s \\
& -\left(f^{\prime}(u(t)) \partial_{t} u(t), \partial_{t} u(t)\right)-\delta\left|\partial_{t} u(t)\right|^{2}, \quad t>0 .
\end{aligned}
$$

Due to (6.5), the integral term in the right-hand side of (6.11) is negative. Using (6.7), we clearly have

$$
\begin{aligned}
& \left|\nabla \partial_{t} u(t)\right|^{2}+\left(f^{\prime}(u(t)) \partial_{t} u(t), \partial_{t} u(t)\right)+\delta\left|\partial_{t} u(t)\right|^{2} \\
& \quad \geq \lambda_{1}\left|\partial_{t} u(t)\right|^{2}-D\left|\partial_{t} u(t)\right|^{2}+\delta\left|\partial_{t} u(t)\right|^{2} \\
& \quad=\rho\left|\partial_{t} u(t)\right|^{2}, \quad \forall t>0,
\end{aligned}
$$


where $\rho=\lambda_{1}-D+\delta$. Hence, using (6.6), we obtain

$$
\frac{d}{d t} J(S(t) v) \leq-\rho\left|\partial_{t} u(t)\right|^{2}, \quad \forall t>0 .
$$

Integrating (6.12) in $t$, we deduce, for the Faedo-Galerkin approximation $u_{N}(t)=$ $u(t)$, the inequality

$$
J\left(S\left(t_{2}\right) v_{N}\right)-J\left(S\left(t_{1}\right) v_{N}\right) \leq-\rho \int_{t_{1}}^{t_{2}}\left|\partial_{t} u_{N}(\tau)\right|^{2} d \tau, \quad \forall t_{2}>t_{1}>0
$$

We now briefly explain the passage to the limit in (6.13) as $N \rightarrow \infty$. We rewrite this inequality as follows:

$$
J\left(S\left(t_{2}\right) v_{N}\right)+\rho \int_{t_{1}}^{t_{2}}\left|\partial_{t} u_{N}(\tau)\right|^{2} d \tau \leq J\left(S\left(t_{1}\right) v_{N}\right), \quad \forall t_{2}>t_{1}>0 .
$$

Notice that the exact solution $u(\cdot)$ of $(2.1)-(2.2)$ belongs to $C_{b}\left(\left[t_{1}, \infty\right) ; H_{0}^{1}(\Omega) \cap\right.$ $\left.L_{p}(\Omega)\right)$ and that $\partial_{t} u(\cdot) \in L_{2}^{b}\left(\left[t_{1}, \infty\right) ; H_{0}^{1}(\Omega)\right)$. Consider an initial datum of the form

$$
\left.\bar{u}\right|_{t \leq t_{1}}=u(t)
$$

for problem (2.1)-(2.2), with an unknown function $\bar{u}, t \geq t_{1}$. Then, we construct the corresponding Faedo-Galerkin approximation $\bar{u}_{N}(\cdot)$. Using the uniqueness of the solution of the Cauchy problem (2.1), (2.2), and (2.17), we observe that $\bar{u}_{N}(\cdot)$ converges to $u(\cdot)$ as $N \rightarrow \infty$ in the corresponding spaces. Since the initial datum is smooth at $t=t_{1}$, it follows that $\bar{u}_{N}(\cdot)-u(\cdot)(N \rightarrow \infty) *$-weakly in the space $L_{\infty}\left(\left[t_{1}, t_{2}\right] ; H_{0}^{1}(\Omega) \cap L_{p}(\Omega)\right)$ and $\partial_{t} \bar{u}_{N}(\cdot) \rightarrow \partial_{t} u(\cdot)(N \rightarrow \infty) *$-weakly in $L_{2}\left(\left[t_{1}, t_{2}\right] ; L_{2}(\Omega)\right)$. Moreover, $J\left(S\left(t_{1}\right) \bar{u}_{N}\right) \rightarrow J\left(S\left(t_{1}\right) u\right)(N \rightarrow \infty)$ in $\mathbb{R}$.

We now pass to the limit in (6.14) as $N \rightarrow \infty$ by using the following known result on weak convergence.

Lemma 6.2. Let $Y$ be a Banach space and $\left\{y_{n}\right\} \subset Y$, with $y_{n}-y$ as $n \rightarrow \infty$, weakly in $Y$. Then, $\|y\|_{Y} \leq \liminf _{n \rightarrow \infty}\left\|y_{n}\right\|_{Y}$. Similarly, if $\left\{f_{n}\right\} \subset Y^{*}$ and $f_{n}-f$ as $n \rightarrow \infty$, *-weakly in $Y$, then $\|f\|_{Y^{*}} \leq \liminf _{n \rightarrow \infty}\left\|f_{n}\right\|_{Y^{*}}$ (see, for instance, [19]).

We now use the Lyapunov function constructed above in order to study the structure of the trajectory and global attractors of the heat equation with memory (2.1).

Corollary 6.3. If $D<\lambda_{1}+\delta$, then any function $v \in \mathfrak{A}$ satisfies the inequality

$$
\int_{-\infty}^{0}\left|\partial_{t} v(s)\right|^{2} d s \leq C_{8}
$$


Here, the constant $C_{8}$ is independent of $v$. Furthermore, concerning the corresponding solution $u(\cdot)$ of problem (2.1), (2.2), and (2.17), we have

$$
\int_{-\infty}^{\infty}\left|\partial_{t} u(s)\right|^{2} d s \leq C_{8} .
$$

To prove (6.15) and (6.16), we note that the trajectory attractor $\mathfrak{A}$ is bounded in the space $L_{\infty}\left(\mathbb{R}_{-} ; H_{0}^{1}(\Omega) \cap L_{p}(\Omega)\right)$ and that the set $\left\{\partial_{t} v \mid v \in \mathfrak{A}\right\}$ is bounded in $L_{\infty}\left(\mathbb{R}_{-} ; L_{2}(\Omega)\right)$ (see Corollary 5.2). Therefore, the functional $J(\cdot)$ is bounded on $\mathfrak{A}$. Furthermore, for any $v \in \mathfrak{A}$, the function $v(t), t \leq 0$, satisfies (2.1) for $t \leq 0$ and (6.8) implies

$$
\rho \int_{t_{1}}^{t_{2}}\left|\partial_{t} u(\tau)\right|^{2} d \tau \leq J\left(S\left(t_{1}\right) v\right)-J\left(S\left(t_{2}\right) v\right), \quad \forall t_{1}, t_{2} \in \mathbb{R}, t_{2} \geq t_{1} .
$$

Here, as usual, $(S(t) v)(s)=u(t+s)$, where $s \leq 0, t \in \mathbb{R}$, and $u(t)$ is the solution of problem (2.1), (2.2), and (2.17), with initial datum $v \in \mathfrak{A}$. The right-hand side of (6.17) is bounded from above by a constant $C_{8}$ independent of $v, t_{1}$, and $t_{2}$. Setting $t_{2}=0$ and passing to the limit as $t_{1} \rightarrow-\infty$, we obtain (6.15). Inequality (6.16) follows from (6.15), since $\mathfrak{A}$ is a strictly invariant set w.r.t. $\{S(t)\}$.

We now prove that the trajectory and global attractors of the heat equation with memory (2.1) have a regular structure when $D<\lambda_{1}+\delta$.

We first go back to the general equation with memory (1.1) considered in Section 1 and give some new important definitions.

We denote by $\mathcal{N}$ the set of the stationary points of the semigroup $\{S(t)\}$ corresponding to equation (1.1) and acting in the space $\mathcal{E}^{-} \subseteq C_{b}\left(\mathbb{R}_{-} ; E\right)$, where $E$ is a Banach space, that is,

$$
z \in \mathcal{N} \Longleftrightarrow S(t) z=z, \quad \forall t \geq 0 .
$$

It is clear that any function $z$ belonging to $\mathcal{N}$ is independent of the time, i.e., $z(s)=z(0)$ for all $s \leq 0$, and satisfies the equation $A(z, z)=0$.

Recall that the bounded sets in $\mathcal{E}^{-}$are defined by considering a Banach space $\mathcal{F} \supseteq \mathcal{E}^{-}$.

For any subset $\mathcal{N}^{\prime} \subseteq \mathcal{N}$, we set

$$
\begin{array}{r}
\mathcal{M}^{+}\left(\mathcal{N}^{\prime}\right)=\left\{v(\cdot) \in \mathcal{E}^{-} \mid v(t) \text { satisfies }(1.1) \text { for } t \leq 0,\right. \\
\text { and } \left.\operatorname{dist}_{E}\left(v(t), \mathcal{N}^{\prime}\right) \rightarrow 0(t \rightarrow-\infty)\right\} .
\end{array}
$$

In other words, the set $\mathcal{M}^{+}\left(\mathcal{N}^{\prime}\right)$ consists of all the solutions of equation (1.1) for negative $t$ which converge to $\mathcal{N}^{\prime}$ as $t \rightarrow-\infty$.

The set $\mathcal{M}^{+}\left(\mathcal{N}^{\prime}\right)$ is called the unstable trajectory set issuing from $\mathcal{N}^{\prime}$. In particular, we can consider the unstable trajectory set $\mathcal{M}^{+}(z)$ issuing from the 
stationary point $z \in \mathcal{N}$ :

$\mathcal{M}^{+}(z)=\left\{v(\cdot) \in \mathcal{E}^{-} \mid v(t)\right.$ satisfies (1.1) for $t \leq 0$, and $\left.\|v(t)-z\|_{E} \rightarrow 0(t \rightarrow-\infty)\right\}$.

We note that the set $\mathcal{M}^{+}\left(\mathcal{N}^{\prime}\right)$ is strictly invariant with respect to $\{S(t)\}$, i.e.,

$$
S(t) \mathcal{M}^{+}\left(\mathcal{N}^{\prime}\right)=\mathcal{M}^{+}\left(\mathcal{N}^{\prime}\right), \quad \forall t \geq 0 .
$$

Remark 6.4. If the set $\mathcal{N}$ is finite, i.e., $\mathcal{N}=\left\{z_{1}, z_{2}, \ldots, z_{m}\right\}$, then

$$
\mathcal{M}^{+}(\mathcal{N})=\bigcup_{j=1}^{m} \mathcal{M}^{+}\left(z_{j}\right) .
$$

Proposition 6.5. If the semigroup $\{S(t)\}$ corresponding to the equation with memory $(1.1)$ possesses the trajectory $\left(\mathcal{F}, C^{\operatorname{loc}}\left(\mathbb{R}_{-} ; E\right)\right)$-attractor $\mathfrak{A}$, then

$$
\begin{aligned}
\mathcal{M}^{+}(\mathcal{N}) & \subseteq \mathfrak{A}, \\
\mathcal{M}^{+}(\mathcal{N})(0) & \subseteq \mathcal{A},
\end{aligned}
$$

where $\mathcal{A}$ is the global $(\mathcal{F}, E)$-attractor of $(1.1)$.

Proof. If $v \in \mathcal{M}^{+}(\mathcal{N})$, then the set $B_{0}=\{T(t) v \mid t \in \mathbb{R}\}, T$ being the translation group, is bounded in $\mathcal{F}$ and strictly invariant w.r.t. $\{S(t)\}$, i.e., $S(t) B_{0}=B_{0}$ for all $t \geq 0$. Therefore, since $S(t) B_{0} \rightarrow \mathfrak{A}$ as $t \rightarrow \infty$, we obtain that $B_{0} \subseteq \mathfrak{A}$, that is, $v \in \mathfrak{A}$ for all $v \in \mathcal{M}^{+}(\mathcal{N})$.

We now return to the heat equation with memory (2.1), for which

$$
\mathcal{E}^{-}=\mathcal{F}=C_{b}\left(\mathbb{R}_{-} ; L_{2}(\Omega)\right) \cap L_{2}^{b}\left(\mathbb{R}_{-} ; H_{0}^{1}(\Omega)\right) .
$$

Let $\mathcal{N}$ be the set of the stationary points of (2.1)-(2.2). The set $\mathcal{N}$ consists obviously of the functions $z \in H_{0}^{1}(\Omega)$ such that

$$
(1+\alpha) \Delta z(x)-f(z(x))+g(x)=0,\left.\quad z\right|_{\partial \Omega}=0,
$$

where $\alpha=\int_{0}^{\infty} k(s) d s$. Consider the corresponding unstable set $\mathcal{M}^{+}(\mathcal{N})$ issuing from $\mathcal{N}$. We are interested in knowing when, in (6.19) and (6.20), the equalities hold in place of the inclusions.

Theorem 6.6. Under condition (6.7), we have

$$
\begin{aligned}
\mathfrak{A} & =\mathcal{M}^{+}(\mathcal{N}), \\
\mathcal{A} & =\mathcal{M}^{+}(\mathcal{N})(0) .
\end{aligned}
$$


Proof. Consider any fixed function $v \in \mathfrak{A}$. We claim that

$$
\operatorname{dist}_{L_{2}(\Omega)}(v(t), \mathcal{N}) \rightarrow 0 \quad(t \rightarrow-\infty) .
$$

Assume the converse. Then, there exist a number $\varepsilon>0$ and a negative sequence $\left\{t_{n}\right\}, t_{n} \rightarrow-\infty(n \rightarrow \infty)$, such that, for all $n \in \mathbb{N}$,

$$
\operatorname{dist}_{L_{2}(\Omega)}\left(v\left(t_{n}\right), \mathcal{N}\right) \geq \varepsilon>0 .
$$

Consider the functions $v_{n}(s)=v\left(t_{n}+s\right), n \in \mathbb{N}$. It is clear that $v_{n}(\cdot) \in \mathfrak{A}$. The set $\mathfrak{A}$ is compact in $C^{\mathrm{loc}}\left(\mathbb{R}_{-} ; L_{2}(\Omega)\right)$. Therefore, by refining, we can assume that the sequence $\left\{v_{n}(\cdot)\right\}$ converges to a function $z(\cdot) \in \mathfrak{A}$ in $C^{\text {loc }}\left(\mathbb{R}_{-} ; L_{2}(\Omega)\right)$. Let us show that $z(s)=z(0)$ for all $s \leq 0$. We fix an arbitrary number $M>0$. It follows from Corollary 4.4 that the sequence $\left\{\partial_{t} v_{n}(\cdot)\right\}$ is bounded in $L_{2}\left(-M, 0 ; L_{2}(\Omega)\right)$ and, therefore, $\partial_{t} v_{n}-\partial_{t} z(n \rightarrow \infty)$ weakly in $L_{2}\left(-M, 0 ; L_{2}(\Omega)\right)$. Hence, Lemma 6.2 implies that

$$
\left\|\partial_{t} z\right\|_{L_{2}\left(-M, 0 ; L_{2}(\Omega)\right)}^{2} \leq \lim \inf _{n \rightarrow \infty}\left\|\partial_{t} v_{n}\right\|_{L_{2}\left(-M, 0 ; L_{2}(\Omega)\right)}^{2} .
$$

We note that

$$
\left\|\partial_{t} v_{n}\right\|_{L_{2}\left(-M, 0 ; L_{2}(\Omega)\right)}^{2}=\int_{t_{n}-M}^{t_{n}}\left|\partial_{t} v(s)\right|^{2} d s .
$$

Then, inequality (6.15) yields that the right-hand side of (6.27) approaches zero as $t_{n} \rightarrow-\infty$. Therefore, $\liminf \operatorname{in}_{n \rightarrow \infty}\left\|\partial_{t} v_{n}\right\|_{L_{2}\left(-M, 0 ; L_{2}(\Omega)\right)}^{2}=0$ and, by (6.26), $\left\|\partial_{t} z\right\|_{L_{2}\left(-M, 0 ; L_{2}(\Omega)\right)}^{2}=0$, that is, $z(s)=z(0)$ for all $s \in[-M, 0]$. The number $M$ was arbitrary so far. It thus follows that $z$ is independent of the time and $z \in \mathcal{N}$. Since $v_{n} \rightarrow z(n \rightarrow \infty)$ in $C\left([-M, 0] ; L_{2}(\Omega)\right)$, we conclude that

$$
\operatorname{dist}_{L_{2}(\Omega)}\left(v\left(t_{n}\right), \mathcal{N}\right)=\operatorname{dist}_{L_{2}(\Omega)}\left(v_{n}(0), \mathcal{N}\right) \rightarrow 0 \quad(n \rightarrow \infty) .
$$

This contradicts inequality (6.25). Consequently, (6.24) holds and we have proved (6.22) and (6.23).

Corollary 6.7. If the set $\mathcal{N}$ is finite and $D<\lambda_{1}+\delta$, then

$$
\begin{aligned}
\mathfrak{A} & =\bigcup_{j=1}^{m} \mathcal{M}^{+}\left(z_{j}\right), \\
\mathcal{A} & =\bigcup_{j=1}^{m} \mathcal{M}^{+}\left(z_{j}\right)(0) .
\end{aligned}
$$

Similarly to Theorem 6.6 , we prove the following property on the solutions of equation (2.1). 
Proposition 6.8. If the nonlinear term $f(u)$ satisfies (6.7), then, for any solution $u(t)$ of problem (2.1), (2.2), and (2.17),

$$
\operatorname{dist}_{L_{2}(\Omega)}(u(t), \mathcal{N}) \rightarrow 0 \quad(t \rightarrow \infty) .
$$

In particular, if $\mathcal{N}$ is finite, then there exists a stationary point $z_{j} \in \mathcal{N}$ such that

$$
\left|u(t)-z_{j}\right| \rightarrow 0 \quad(t \rightarrow \infty) .
$$

Remark 6.9. In fact, for any $v \in \mathfrak{A}, v(t) \rightarrow \mathcal{N}$ as $t \rightarrow-\infty$ in $H^{1-\varepsilon}(\Omega)$ and any solution $u(t)$ of (2.1)-(2.2) converges to $\mathcal{N}$ as $t \rightarrow+\infty$ in the norm of $H^{1-\varepsilon}(\Omega)$, where $\varepsilon \in(0,1]$. This obvious improvement follows from Corollary 4.5.

To conclude, we note that, if the nonlinear function $f(u)$ satisfies the inequality

$$
-f^{\prime}(u) \leq D<\lambda_{1}(1+\alpha),
$$

then the set $\mathcal{N}$ consists of a unique stationary point $z_{0}$ (see, for instance, [13]) and, therefore, any solution $u(t)$ of the heat equation with memory (2.1) converges to $z_{0}$ as $t \rightarrow+\infty$. Thus, in that case, the trajectory and global attractors associated with equation (2.1) are trivial.

The picture becomes more complicated when

$$
\lambda_{1}(1+\alpha)<D<\lambda_{1}+\delta .
$$

Examples show that, in that case, the set $\mathcal{N}$ may consist in more than one stationary point (for instance, the functions $f(u)=-D u+\alpha u^{3}$ and $g \equiv 0$ give the Chafee-Infante equation with memory) and the attractors are still regular and coincide with the corresponding unstable sets issuing from $\mathcal{N}$.

The question "is the trajectory attractor of the heat equation with memory and a general nonlinear function $f(u)$ regular?" remains open, since we do not know how to construct a Lyapunov function for the heat equation with memory in general.

Acknowledgement. This work was partially supported by INTAS, Grant no. 00-899, the Russian Foundation for Fundamental Research, Project no. 02-0100227, and the Civilian Research \& Development Foundation (CRDF), Grant no. RM1-2343-MO-02.

\section{REFERENCES}

[1] Anatoli V. Babin and Mark I. VishiK, Attraktory evolyutsionnykh uravnenii, Nauka, Moscow, 1989, ISBN 5-02-006632-X. MR1007829 (92f:58101) (Russian)

[2] Vladimir V. Chepyzhov and Mark I. VishiK, Attractors for Equations of Mathematical Physics, American Mathematical Society Colloquium Publications, vol. 49, American Mathematical Society, Providence, RI, 2002, ISBN 0-8218-2950-5. MR1868930 (2003f:37001c) 
[3] _ Evolution equations and their trajectory attractors, J. Math. Pures Appl. (9) 76 (1997), 913-964. MR1489945 (99a:34165)

[4] Vladimir V. Chepyzhov and Alain Miranville, Trajectory and global attractors of dissipative hyperbolic equations with memory, Commun. Pure Appl. Anal. 4 (2005), 115-142. MR2126281 (2005k:37178)

[5] Bernard D. Coleman and Morton E. Gurtin, Equipresence and constitutive equations for rigid heat conductors, Z. Angew. Math. Phys. 18 (1967), 199-208. MR0214334 (35 \#5185) (English, with German summary)

[6] Constantine M. Dafermos, Asymptotic stability in viscoelasticity, Arch. Rational Mech. Anal. 37 (1970), 297-308, http://dx.doi.org/10.1007/BF00251609. MR0281400 (43 \#7117)

[7] Claudio Giorgi, Maria Grazia Naso, and VitTorino Pata, Exponential stability in linear heat conduction with memory: a semigroup approach, Commun. Appl. Anal. 5 (2001), 121133. MR1844676 (2002e:35233)

[8] Claudio Giorgi and Vittorino Pata, Asymptotic behavior of a nonlinear hyperbolic heat equation with memory, NoDEA Nonlinear Differential Equations Appl. 8 (2001), 157-171. MR1834457 (2002d:35210)

[9] Claudio Giorgi, Vittorino Pata, and Alfredo Marzocchi, Asymptotic behavior of a semilinear problem in heat conduction with memory, NoDEA Nonlinear Differential Equations Appl. 5 (1998), 333-354, http://dx.doi.org/10.1007/s000300050049. MR1638908 (99h:35081)

[10] Uniform attractors for a non-autonomous semilinear heat equation with memory, Quart. Appl. Math. 58 (2000), 661-683. MR1788423 (2001k:37129)

[11] Morton E. Gurtin and Allen C. Pipkin, A general theory of heat conduction with finite wave speed, Arch. Rational Mech. Anal. 31 (1968), 113-126.

[12] JACK K. Hale, Asymptotic Behavior of Dissipative Systems, Mathematical Surveys and Monographs, vol. 25, American Mathematical Society, Providence, RI, 1988, ISBN 0-8218-1527-X. MR941371 (89g:58059)

[13] Daniel Henry, Geometric Theory of Semilinear Parabolic Equations, Lecture Notes in Mathematics, vol. 840, Springer-Verlag, Berlin, 1981, ISBN 3-540-10557-3. MR610244 (83j:35084)

[14] JACQUES-LOUIS LIONS, Quelques méthodes de résolution des problèmes aux limites non linéaires, Dunod, Paris, 1969. MR0259693 (41 \#4326) (French)

[15] JACQUes-LOUIS LiONS and ENRICO MAgenes, Problèmes aux limites non homogènes et applications, vol. Vol.1, Dunod, Paris, 1968. MR0247243 (40 \#512)

[16] Josef Meixner, On the linear theory of heat conduction, Arch. Rat. Mech. Anal. 39 (1970), 108, http://dx.doi.org/10.1007/BF00281042.

[17] JACE W. NunZIATO, On heat conduction in materials with memory, Quart. Appl. Math. 29 (1971), 187-204. MR0295683 (45 \#4749)

[18] VitToRino PATA, Hyperbolic limit of parabolic semilinear heat equations with fading memory, Z. Anal. Anwendungen 20 (2001), 359-377. MR1846607 (2002i:35093)

[19] Roger Temam, Infinite-dimensional Dynamical Systems in Mechanics and Physics, Applied Mathematical Sciences, vol. 68, Springer-Verlag, New York, 1997, ISBN 0-387-94866-X. MR1441312 (98b:58056)

[20] __ Navier-Stokes Equations. Theory and Numerical Analysis, North-Holland Publishing Co., Amsterdam, 1977, ISBN 0-7204-2840-8. MR0609732 (58 \#29439) 
V.V. Chepyzhov:

Institute for Problems of Information Transmission

Russian Academy of Sciences, Bolshoy Karetniy 19

Moscow 127994, GSP-4, Russia.

E-MAIL: chep@iitp.ru

A. MiranVille:

Université de Poitiers

Laboratoire de Mathématiques et Applications

UMR 6086 - CNRS, SP2MI

Boulevard Marie et Pierre Curie - Téléport 2

86962 Chasseneuil Futuroscope Cedex, France.

E-MAIL: miranv@mathlabo.univ-poitiers.fr

KEY WORDS AND PHRASES:

Heat equation with memory; trajectory attractor; global attractor; Lyapunov function. 2000 Mathematics Subject Classification: 33B41, 37L30, 45 K05.

Received: January 30th, 2004; revised: June 14th, 2004. 